\title{
THEORETICAL ANALYSIS OF SINC-NYSTRÖM METHODS FOR VOLTERRA INTEGRAL EQUATIONS
}

\author{
TOMOAKI OKAYAMA, TAKAYASU MATSUO, AND MASAAKI SUGIHARA
}

\begin{abstract}
In this paper, we present three theoretical results on Sinc-Nyström methods for Volterra integral equations of the first and second kind, which were proposed by Muhammad et al. Their methods involve the following issues: 1) it is difficult to determine the tuning parameter unless the solution is given, and 2) convergence has not been proved in a precise sense. In a mathematically rigorous manner, we present an implementable way to estimate the tuning parameter and a rigorous proof of the convergence with its rate explicitly revealed. Furthermore, we show that the resulting system is well conditioned. Numerical examples that support the theoretical results are also presented.
\end{abstract}

\section{INTRODUCTION}

We are mainly concerned with Volterra integral equations of the second kind,

$$
u(t)-\int_{a}^{t} k(t, s) u(s) \mathrm{d} s=g(t), \quad a \leq t \leq b,
$$

where $g(t)$ and $k(t, s)$ are given functions, and $u(t)$ is the solution to be determined. These equations have been employed for mathematical modeling in many fields, such as renewal processes [3], semi-conductor devices [12, wave phenomena [7, and population biology [4. Owing to their significance in such applications, numerous studies have investigated their theoretical aspects and numerical methods (see, for example, Linz [11], Kythe-Puri [9], Brunner [5], and the references therein). Among these numerical methods, discretization by collocation method is usually employed. The typical procedure is as follows. Set an approximate solution $u_{n}$ in a linear combination of some basis functions $\omega_{j}(t)$ as $u_{n}(t)=\sum_{j=1}^{n} c_{j} \omega_{j}(t)$ and substitute $u_{n}$ into (1.1) to obtain a system of linear equations

$$
u_{n}\left(t_{i}\right)-\int_{a}^{t_{i}} k\left(t_{i}, s\right) u_{n}(s) \mathrm{d} s=g\left(t_{i}\right), \quad i=1, \ldots, n,
$$

where $t_{i}(i=1, \ldots, n)$ are the collocation points. Unknown coefficients $c_{j}$ are obtained by solving this system, and the approximate solution $u_{n}$ is determined.

If we turn our attention to Fredholm integral equations of the second kind,

$$
u(t)-\int_{a}^{b} k(t, s) u(s) \mathrm{d} s=g(t), \quad a \leq t \leq b,
$$

a strong alternative to the collocation method is Nyström's method 2, 8, 9. The typical procedure is as follows. Let a quadrature rule be given as $\int_{a}^{b} f(s) \mathrm{d} s \approx$

Received by the editor March 26, 2013.

2010 Mathematics Subject Classification. Primary 65R20; Secondary 45D05.

The first author was supported by JSPS Grants-in-Aid for Scientific Research. 
$\sum_{j=1}^{n} w_{j} f\left(t_{j}\right)$. Using this rule, the integral in (1.2) may be approximated as

$$
u_{n}(t)=g(t)+\sum_{j=1}^{n} w_{j} k\left(t, t_{j}\right) u_{n}\left(t_{j}\right), \quad a \leq t \leq b .
$$

Here, we see that the approximate solution $u_{n}$ is determined if we find the values $u_{n}\left(t_{j}\right)(j=1, \ldots, n)$. These values are obtained by solving the system of linear equations

$$
u_{n}\left(t_{i}\right)=g\left(t_{i}\right)+\sum_{j=1}^{n} w_{j} k\left(t_{i}, t_{j}\right) u_{n}\left(t_{j}\right), \quad i=1, \ldots, n,
$$

derived by sampling (1.3) at $t=t_{i}$.

In contrast, the Nyström method does not work for Volterra integral equations (1.1) because the interval of the integral is indefinite: $[a, t]$. If we use the quadrature rule above, we must adjust the weight $w_{j}$ and quadrature node $t_{j}$ with respect to $t$ as $\int_{a}^{t} f(s) \mathrm{d} s \approx \sum_{j=1}^{n} w_{j}(t) f\left(t_{j}(t)\right)$. This dependency on $t$ causes a problem; (1.3) becomes

$$
u_{n}(t)=g(t)+\sum_{j=1}^{n} w_{j}(t) k\left(t, t_{j}(t)\right) u_{n}\left(t_{j}(t)\right), \quad a \leq t \leq b,
$$

which does not lead to a linear system such as (1.4), at least in the same way. Therefore, the Nyström method has not been generally considered appropriate for Volterra integral equations.

In opposition to such a general view, Muhammad et al. 14 dared to employ the Nyström method to develop a numerical scheme for Volterra integral equations (1.1). Instead of a quadrature rule, they employed an indefinite integration rule called "Sinc indefinite integration" in order to approximate the indefinite integral, which can be expressed as $\int_{a}^{t} f(s) \mathrm{d} s \approx \sum_{j=-N}^{N} \tilde{w}_{j}(t) f\left(s_{j}\right)$, where the node point $s_{j}$ is fixed. As a result, (1.5) now becomes

$$
u_{N}(t)=g(t)+\sum_{j=-N}^{N} \tilde{w}_{j}(t) k\left(t, s_{j}\right) u_{N}\left(s_{j}\right), \quad a \leq t \leq b,
$$

which naturally derives the linear system

$$
u_{N}\left(s_{i}\right)=g\left(s_{i}\right)+\sum_{j=-N}^{N} \tilde{w}_{j}\left(s_{i}\right) k\left(s_{i}, s_{j}\right) u_{N}\left(s_{j}\right), \quad i=-N, \ldots, N,
$$

by sampling (1.6) at $t=s_{i}$. This procedure is called the "Sinc-Nyström method" (details are described in Section 3).

Furthermore, the work by Muhammad et al. 14 had another beautiful property that exponential convergence $\mathrm{O}(\exp (-c N / \log N))$ can be numerically observed. They also gave an error analysis of the form (see Theorem 3.1)

$$
\sup _{t \in(a, b)}\left|u(t)-u_{N}(t)\right| \leq\left(C \sqrt{N}\left\|A_{N}^{-1}\right\|_{2}+C^{\prime}\right) \frac{\log N}{N} \exp (-c N / \log N)
$$


where $A_{N}$ denotes the coefficient matrix of the resulting linear system (1.7). The existence of $A_{N}^{-1}$ and stability of its norm as $N \rightarrow \infty$ were not proved, but suggested by their numerical experiments.

One of the objectives of this study is to reinforce their scheme by presenting a complete error analysis of the form (see Theorem 3.5)

$$
\max _{t \in[a, b]}\left|u(t)-u_{N}(t)\right| \leq C \frac{\log N}{N} \exp (-c N / \log N)
$$

which does not include any unestimated term, and thus, rigorously proves the exponential convergence of the solution $u_{N}$. In the Fredholm case (1.2), the present authors [16] developed an analysis technique for the Sinc-Nyström method, where the "collectively compact" operator theory [1] was utilized. In the Volterra case (1.1), however, the same strategy does not work because it is hard (presumably impossible) to show that the approximated operator obtained by means of the Sinc indefinite integration is collectively compact. In this sense, there is quite a big gap between the Fredholm and Volterra type integral equations. In order to overcome this difficulty, we take a different approach (details are given in Section 66). Furthermore, we reinforce the scheme of Muhammad et al. 14] by proving the existence of $A_{N}^{-1}$ and the boundedness of both $\left\|A_{N}\right\|_{\infty}$ and $\left\|A_{N}^{-1}\right\|_{\infty}$, implying that the resulting system is well conditioned.

Another objective of this study, which is more important in practice, is to introduce a way to estimate a tuning parameter " $d$ ", which is required to set the mesh size $h$ (see (2.5) and (2.6). The choice is important since it substantially affects the convergence profile of their schemes (see the numerical examples in Section 4.2). However, the optimal value of this parameter is, in principle, determined from the unknown true solution $u$, and thus far, no practical way to find the optimal value has been found. As a remedy, we theoretically show that the parameter can be estimated by investigating known functions $k$ and $g$, instead of the solution $u$. Similar results have already been given for Fredholm integral equations [15, 16, 22, but these are the first reported for Volterra integral equations.

The remainder of this paper is organized as follows. Section 2 is a preliminary section that states numerical indefinite integration formulas by means of the Sinc approximation and their convergence theorems. In Section 3, we review the schemes and error analysis presented by Muhammad et al. 14, and we summarize new theoretical analyses (main results). The corresponding proofs are given in Sections 567. Numerical experiments are described in Section 4, where the focus is mainly on the tuning parameter $d$. In addition to the equations of the second kind (1.1), Muhammad et al. 14] also considered equations of the first kind,

$$
\int_{a}^{t} k(t, s) u(s) \mathrm{d} s=g(t), \quad a \leq t \leq b, \quad g(a)=0,
$$

by simply rewriting them as second kind equations. Although this approach might not be as attractive as existing, elegant, direct approaches (see, for example, Lamm [10]), we also state our theoretical results for it in Section 8 in order to cover all cases considered in Muhammad et al. 14. Finally, Section 9 concludes this paper. 


\section{Preliminary: Sinc indefinite integration}

2.1. Sinc indefinite integration on the real axis. Let us first consider the approximation on the entire real axis. A function approximation expressed as

$$
F(\sigma) \approx \sum_{j=-N}^{N} F(j h) S(j, h)(\sigma), \quad \sigma \in \mathbb{R},
$$

is called the "Sinc approximation," where the basis function $S(j, h)$ is defined by

$$
S(j, h)(x)=\frac{\sin [\pi(x / h-j)]}{\pi(x / h-j)} .
$$

The mesh size $h$ should be appropriately selected depending on $N$ and smoothness of the function $F$ (described in the subsequent convergence theorems). Using the Sinc approximation, Haber [6] derived the numerical indefinite integration formula

$$
\begin{aligned}
\int_{-\infty}^{x} F(\sigma) \mathrm{d} \sigma & \approx \sum_{j=-N}^{N} F(j h) \int_{-\infty}^{x} S(j, h)(\sigma) \mathrm{d} \sigma \\
& =\sum_{j=-N}^{N} F(j h) J(j, h)(x), \quad x \in \mathbb{R},
\end{aligned}
$$

where the basis function $J(j, h)$ is defined by

$$
J(j, h)(x)=h\left\{\frac{1}{2}+\frac{1}{\pi} \operatorname{Si}[\pi(x / h-j)]\right\} .
$$

Here, $\operatorname{Si}(x)$ is the so-called "sine integral" function, whose routine is available in some numerical libraries (IMSL, NAG, GSL, and so on). We call formula (2.1) the "Sinc indefinite integration."

2.2. SE-Sinc indefinite integration and DE-Sinc indefinite integration. In the case of the finite interval (recall the target equations (1.1) and (1.8)), variable transformation is often utilized. The standard transformation is the "SingleExponential transformation" defined by

$$
s=\psi^{\mathrm{SE}}(\sigma)=\frac{b-a}{2} \tanh \left(\frac{\sigma}{2}\right)+\frac{b+a}{2},
$$

which maps $\sigma \in \mathbb{R}$ onto $s \in(a, b)$. We call it the "SE transformation" for short. The inverse function is

$$
\sigma=\left\{\psi^{\mathrm{SE}}\right\}^{-1}(s)=\log \left(\frac{s-a}{b-s}\right) .
$$

Combining the SE transformation with the Sinc indefinite integration (2.1), Haber [6] derived the following indefinite integration formula on the finite interval:

$$
\begin{aligned}
\int_{a}^{t} f(s) \mathrm{d} s & =\int_{-\infty}^{\left\{\psi^{\mathrm{SE}}\right\}^{-1}(t)} f\left(\psi^{\mathrm{SE}}(\sigma)\right)\left\{\psi^{\mathrm{SE}}\right\}^{\prime}(\sigma) \mathrm{d} \sigma \\
& \approx \sum_{j=-N}^{N} f\left(\psi^{\mathrm{SE}}(j h)\right)\left\{\psi^{\mathrm{SE}}\right\}^{\prime}(j h) J(j, h)\left(\left\{\psi^{\mathrm{SE}}\right\}^{-1}(t)\right), \quad t \in[a, b] .
\end{aligned}
$$

We call this approximation the "SE-Sinc indefinite integration." 
In order to improve the convergence rate, Muhammad-Mori 13 proposed replacing the SE transformation with the Double-Exponential (DE) transformation

$$
s=\psi^{\mathrm{DE}}(\sigma)=\frac{b-a}{2} \tanh \left(\frac{\pi}{2} \sinh (\sigma)\right)+\frac{b+a}{2},
$$

whose inverse function is

$$
\sigma=\left\{\psi^{\mathrm{DE}}\right\}^{-1}(s)=\log \left[\frac{1}{\pi} \log \left(\frac{s-a}{b-s}\right)+\sqrt{1+\left\{\frac{1}{\pi} \log \left(\frac{s-a}{b-s}\right)\right\}^{2}}\right] .
$$

The modified formula is expressed as

$$
\int_{a}^{t} f(s) \mathrm{d} s \approx \sum_{j=-N}^{N} f\left(\psi^{\mathrm{DE}}(j h)\right)\left\{\psi^{\mathrm{DE}}\right\}^{\prime}(j h) J(j, h)\left(\left\{\psi^{\mathrm{DE}}\right\}^{-1}(t)\right), \quad t \in[a, b] .
$$

We call this approximation the "DE-Sinc indefinite integration."

2.3. Convergence theorems. In order to facilitate the error analysis of the formulas stated above, let us introduce the following function spaces.

Definition 2.1. Let $\mathscr{D}$ be a bounded and simply connected domain (or Riemann surface). Then, $\mathbf{H}^{\infty}(\mathscr{D})$ denotes the family of functions $f$ analytic on $\mathscr{D}$ such that the norm $\|f\|_{\mathbf{H}^{\infty}(\mathscr{D})}$ is finite, where

$$
\|f\|_{\mathbf{H}^{\infty}(\mathscr{D})}=\sup _{z \in \mathscr{D}}|f(z)| .
$$

Definition 2.2. Let $\alpha$ be a positive constant, and let $\mathscr{D}$ be a bounded and simply connected domain (or Riemann surface) that satisfies $(a, b) \subset \mathscr{D}$. Then, $\mathbf{L}_{\alpha}(\mathscr{D})$ denotes the family of functions $f \in \mathbf{H}^{\infty}(\mathscr{D})$ for which there exists a constant $K$ such that for all $z$ in $\mathscr{D}$,

$$
|f(z)| \leq K|Q(z)|^{\alpha},
$$

where the function $Q$ is defined by $Q(z)=(z-a)(b-z)$.

In what follows, the domain $\mathscr{D}$ is supposed to be either

$$
\psi^{\mathrm{SE}}\left(\mathscr{D}_{d}\right)=\left\{z=\psi^{\mathrm{SE}}(\zeta): \zeta \in \mathscr{D}_{d}\right\} \quad \text { or } \quad \psi^{\mathrm{DE}}\left(\mathscr{D}_{d}\right)=\left\{z=\psi^{\mathrm{DE}}(\zeta): \zeta \in \mathscr{D}_{d}\right\},
$$

which denotes the domain translated from the strip domain

$$
\mathscr{D}_{d}=\{\zeta \in \mathbb{C}:|\operatorname{Im} \zeta|<d\},
$$

for a positive constant $d$ (see also Tanaka et al. [23, Figures 1 and 5] for the concrete shape of the domains (2.4), where $d=1$ and $(a, b)=(-1,1))$. Then, convergence theorems of the SE/DE-Sinc indefinite integration can be stated as follows.

Theorem 2.3 (Okayama et al. 17, Theorem 2.9]). Let $(f Q) \in \mathbf{L}_{\alpha}\left(\psi^{\mathrm{SE}}\left(\mathscr{D}_{d}\right)\right)$ for $d$ with $0<d<\pi$. Let $N$ be a positive integer, and let $h$ be selected by the formula

$$
h=\sqrt{\frac{\pi d}{\alpha N}} .
$$


Then, there exists a constant $C_{\alpha, d}^{\mathrm{SE}}$ depending only on $\alpha$ and $d$ such that

$$
\begin{aligned}
& \max _{t \in[a, b]}\left|\int_{a}^{t} f(s) \mathrm{d} s-\sum_{j=-N}^{N} f\left(\psi^{\mathrm{SE}}(j h)\right)\left\{\psi^{\mathrm{SE}}\right\}^{\prime}(j h) J(j, h)\left(\left\{\psi^{\mathrm{SE}}\right\}^{-1}(t)\right)\right| \\
& \leq K(b-a)^{2 \alpha-1} C_{\alpha, d}^{\mathrm{SE}} \mathrm{e}^{-\sqrt{\pi d \alpha N}},
\end{aligned}
$$

where $K$ is the constant in (2.3).

Theorem 2.4 (Okayama et al. [17, Theorem 2.16]). Let $(f Q) \in \mathbf{L}_{\alpha}\left(\psi^{\mathrm{DE}}\left(\mathscr{D}_{d}\right)\right)$ for $d$ with $0<d<\pi / 2$. Let $N$ be a positive integer, and let $h$ be selected by the formula

$$
h=\frac{\log (2 d N / \alpha)}{N} .
$$

Then, there exists a constant $C_{\alpha, d}^{\mathrm{DE}}$ depending only on $\alpha$ and $d$ such that

$$
\begin{aligned}
& \max _{t \in[a, b]}\left|\int_{a}^{t} f(s) \mathrm{d} s-\sum_{j=-N}^{N} f\left(\psi^{\mathrm{DE}}(j h)\right)\left\{\psi^{\mathrm{DE}}\right\}^{\prime}(j h) J(j, h)\left(\left\{\psi^{\mathrm{DE}}\right\}^{-1}(t)\right)\right| \\
& \leq K(b-a)^{2 \alpha-1} C_{\alpha, d}^{\mathrm{DE}} \frac{\log (2 d N / \alpha)}{N} \exp \left[\frac{-\pi d N}{\log (2 d N / \alpha)}\right]
\end{aligned}
$$

where $K$ is the constant in (2.3).

Remark 2.5. For the SE-Sinc indefinite integration, Haber [6] obtained an error estimate $\mathrm{O}\left(\sqrt{N} \mathrm{e}^{-\pi d \alpha N}\right)$, but it is not sufficient for our purpose (see Remark 6.6). Similarly, the convergence rate of the DE-Sinc indefinite integration has been evaluated as $\mathrm{O}(\exp [-\pi d N / \log (2 d N / \alpha)])$ by Muhammad-Mori [13], but the theorem stated above gives a stronger result.

Remark 2.6. In Theorems 2.3 and 2.4, the constants are stated more explicitly than usual. This is needed for the proofs of Lemmas 6.5 and 6.9. In view of (6.9) and (6.15), we observe that the function $F_{i}(t, z) Q(z)$ cannot be bounded by a constant, and it should depend on $h$ (and accordingly, on $N$ ). We have to address the dependency on $N$, and the estimates in that form are needed for this purpose.

\section{REVIEW OF THE EXISTING RESUlts AND the MAIN RESUlts OF THIS PAPER}

First, in Sections 3.1 and 3.2, we review the schemes and theorem given by Muhammad et al. [14] for Volterra integral equations of the second kind (1.1). Then, we summarize the three main results in Sections 3.3 3.5. We should note here that there are two variants of the Sinc-Nyström methods: the SE-Sinc-Nyström method and the DE-Sinc-Nyström method, corresponding to the variable transformation employed in the scheme.

3.1. SE-Sinc-Nyström method. Let $u \in \mathbf{H}^{\infty}\left(\psi^{\mathrm{SE}}\left(\mathscr{D}_{d}\right)\right)$ and let $k(t, \cdot) Q(\cdot) \in$ $\mathbf{L}_{\alpha}\left(\psi^{\mathrm{SE}}\left(\mathscr{D}_{d}\right)\right)$ for all $t \in[a, b]$. Then, according to Theorem 2.3. the integral in (1.1) can be approximated by the SE-Sinc indefinite integration as

$$
\int_{a}^{t} k(t, s) u(s) \mathrm{d} s \approx \sum_{j=-N}^{N} k\left(t, \psi^{\mathrm{SE}}(j h)\right) u\left(\psi^{\mathrm{SE}}(j h)\right)\left\{\psi^{\mathrm{SE}}\right\}^{\prime}(j h) J(j, h)\left(\left\{\psi^{\mathrm{SE}}\right\}^{-1}(t)\right),
$$


where the mesh size $h$ is selected by (2.5). By this approximation, we have a new equation:

$$
u_{N}^{\mathrm{SE}}(t)=g(t)+\sum_{j=-N}^{N} k\left(t, \psi^{\mathrm{SE}}(j h)\right) u_{N}^{\mathrm{SE}}\left(\psi^{\mathrm{SE}}(j h)\right)\left\{\psi^{\mathrm{SE}}\right\}^{\prime}(j h) J(j, h)\left(\left\{\psi^{\mathrm{SE}}\right\}^{-1}(t)\right) .
$$

In order to determine the approximated solution $u_{N}^{\mathrm{SE}}$, we have to obtain the unknown coefficients

$$
\boldsymbol{u}_{m}^{\mathrm{SE}}=\left[u_{N}^{\mathrm{SE}}\left(\psi^{\mathrm{SE}}(-N h)\right), u_{N}^{\mathrm{SE}}\left(\psi^{\mathrm{SE}}(-(N-1) h)\right), \ldots, u_{N}^{\mathrm{SE}}\left(\psi^{\mathrm{SE}}(N h)\right)\right]^{\mathrm{T}},
$$

where $m=2 N+1$. For this purpose, consider the discretization of (3.1) at the SE-Sinc points $t=t_{i}^{\mathrm{SE}}$, where

$$
t_{i}^{\mathrm{SE}}=\psi^{\mathrm{SE}}(i h), \quad i=-N,-N+1, \ldots, N .
$$

Then, the resulting linear system is written as

$$
\left(I_{m}-V_{m}^{\mathrm{SE}}\right) \boldsymbol{u}_{m}^{\mathrm{SE}}=\boldsymbol{g}_{m}^{\mathrm{SE}},
$$

where $I_{m}$ denotes an $m \times m$ identity matrix and $V_{m}^{\mathrm{SE}}$ is an $m \times m$ matrix whose element at $(i, j)$ is defined by

$$
\left(V_{m}^{\mathrm{SE}}\right)_{i j}=k\left(t_{i}^{\mathrm{SE}}, t_{j}^{\mathrm{SE}}\right)\left\{\psi^{\mathrm{SE}}\right\}^{\prime}(j h) J(j, h)(i h), \quad i, j=-N,-N+1, \ldots, N,
$$
and $\boldsymbol{g}_{m}^{\mathrm{SE}} \in \mathbb{R}^{m}$ is defined by

$$
\boldsymbol{g}_{m}^{\mathrm{SE}}=\left[g\left(t_{-N}^{\mathrm{SE}}\right), g\left(t_{-N+1}^{\mathrm{SE}}\right), \ldots, g\left(t_{N}^{\mathrm{SE}}\right)\right]^{\mathrm{T}} .
$$

By solving (3.4), the approximated solution $u_{N}^{\mathrm{SE}}$ is determined by (3.1).

Although the above scheme was mentioned by Muhammad et al. [14, the authors mainly considered the DE-Sinc-Nyström method described below, and no theoretical analysis was given for the above scheme (the SE-Sinc-Nyström method).

3.2. DE-Sinc-Nyström method. Here, we describe the DE-Sinc-Nyström method by replacing the SE transformation used in the SE-Sinc-Nyström method with the DE transformation. Let $u \in \mathbf{H}^{\infty}\left(\psi^{\mathrm{DE}}\left(\mathscr{D}_{d}\right)\right)$ and $k(t, \cdot) Q(\cdot) \in \mathbf{L}_{\alpha}\left(\psi^{\mathrm{DE}}\left(\mathscr{D}_{d}\right)\right)$ for all $t \in[a, b]$. Then, according to Theorem 2.4 the integral in (1.1) can be approximated by the DE-Sinc indefinite integration as

$$
\int_{a}^{t} k(t, s) u(s) \mathrm{d} s \approx \sum_{j=-N}^{N} k\left(t, \psi^{\mathrm{DE}}(j h)\right) u\left(\psi^{\mathrm{DE}}(j h)\right)\left\{\psi^{\mathrm{DE}}\right\}^{\prime}(j h) J(j, h)\left(\left\{\psi^{\mathrm{DE}}\right\}^{-1}(t)\right),
$$

where the mesh size $h$ is selected by (2.6). By this approximation, we have a new equation:

$$
u_{N}^{\mathrm{DE}}(t)=g(t)+\sum_{j=-N}^{N} k\left(t, \psi^{\mathrm{DE}}(j h)\right) u_{N}^{\mathrm{DE}}\left(\psi^{\mathrm{DE}}(j h)\right)\left\{\psi^{\mathrm{DE}}\right\}^{\prime}(j h) J(j, h)\left(\left\{\psi^{\mathrm{DE}}\right\}^{-1}(t)\right) .
$$

In order to determine the approximated solution $u_{N}^{\mathrm{DE}}$, we have to obtain unknown coefficients

$$
\boldsymbol{u}_{m}^{\mathrm{DE}}=\left[u_{N}^{\mathrm{DE}}\left(\psi^{\mathrm{DE}}(-N h)\right), u_{N}^{\mathrm{DE}}\left(\psi^{\mathrm{DE}}(-(N-1) h)\right), \ldots, u_{N}^{\mathrm{DE}}\left(\psi^{\mathrm{DE}}(N h)\right)\right]^{\mathrm{T}},
$$

where $m=2 N+1$. For this purpose, consider the discretization of (3.7) at the DE-Sinc points $t=t_{i}^{\mathrm{DE}}$, where

$$
t_{i}^{\mathrm{DE}}=\psi^{\mathrm{DE}}(i h), \quad i=-N,-N+1, \ldots, N .
$$


Then, the resulting linear system is written as

$$
\left(I_{m}-V_{m}^{\mathrm{DE}}\right) \boldsymbol{u}_{m}^{\mathrm{DE}}=\boldsymbol{g}_{m}^{\mathrm{DE}},
$$

where $V_{m}^{\mathrm{DE}}$ is an $m \times m$ matrix whose element at $(i, j)$ is defined by

$$
\left(V_{m}^{\mathrm{DE}}\right)_{i j}=k\left(t_{i}^{\mathrm{DE}}, t_{j}^{\mathrm{DE}}\right)\left\{\psi^{\mathrm{DE}}\right\}^{\prime}(j h) J(j, h)(i h), \quad i, j=-N,-N+1, \ldots, N,
$$
and $\boldsymbol{g}_{m}^{\mathrm{DE}} \in \mathbb{R}^{m}$ is defined by

$$
\boldsymbol{g}_{m}^{\mathrm{DE}}=\left[g\left(t_{-N}^{\mathrm{DE}}\right), g\left(t_{-N+1}^{\mathrm{DE}}\right), \ldots, g\left(t_{N}^{\mathrm{DE}}\right)\right]^{\mathrm{T}} .
$$

By solving (3.10), the approximated solution $u_{N}^{\mathrm{DE}}$ is determined by (3.7).

For this scheme (DE-Sinc-Nyström method), Muhammad et al. 14 obtained the following error analysis.

Theorem 3.1 (Muhammad et al. 14, Theorem 3.2]). Let $u$ be the solution of (1.1). Let $u \in \mathbf{H}^{\infty}\left(\psi^{\mathrm{DE}}\left(\mathscr{D}_{d}\right)\right)$ and $k(t, \cdot) Q(\cdot) \in \mathbf{L}_{\alpha}\left(\psi^{\mathrm{DE}}\left(\mathscr{D}_{d}\right)\right)$ for all $t \in[a, b]$. Then, there exist constants $C$ and $C^{\prime}$ independent of $N$ such that

$$
\sup _{a<t<b}\left|u(t)-u_{N}^{\mathrm{DE}}(t)\right| \leq\left(C \sqrt{N}\left\|\left(I_{m}-V_{m}^{\mathrm{DE}}\right)^{-1}\right\|_{2}+C^{\prime}\right) \frac{\log N}{N} \exp \left[\frac{-\pi d N}{\log (2 d N / \alpha)}\right] .
$$

3.3. Main result 1: Estimating the tuning parameter $d$. In both the SEand DE-Sinc-Nyström methods, the mesh size $h$ is selected on the basis of the parameters $\alpha$ and $d$ (recall (2.5) and (2.6)). The former parameter $\alpha$ can be obtained by investigating the known function $k$ (recall the assumption in Theorem 3.1). The latter parameter $d$ is, however, not easy to determine because it also depends on the unknown function $u$; not only is it indispensable for launching the schemes, but it also substantially affects the performance (see Section 4.2). Thus far, however, no practical approach for determining the value is known. In the previous study [14, the exact solution $u$ seems to have been used to investigate $d$ for numerical experiments; however, in practical situations, we cannot expect $u$ to be known. To remedy this issue, we present the following result. The proof will be given in Section 5 .

Theorem 3.2. Let $\mathscr{D}=\psi^{\mathrm{SE}}\left(\mathscr{D}_{d}\right)$ or $\mathscr{D}=\psi^{\mathrm{DE}}\left(\mathscr{D}_{d}\right)$. Let $g \in \mathbf{H}^{\infty}(\mathscr{D})$, let $k(z, \cdot) Q(\cdot)$ $\in \mathbf{L}_{\alpha}(\mathscr{D})$, and let $k(\cdot, w) Q(w) \in \mathbf{H}^{\infty}(\mathscr{D})$ for all $z, w \in \mathscr{D}$. Then, (1.1) has a unique solution $u \in \mathbf{H}^{\infty}(\mathscr{D})$.

This theorem states that the parameter $d$ of the unknown function $u$ can be found from known functions $k$ and $g$. Roughly speaking, by investigating the values of $d$ for both $k$ and $g$, we can choose the smaller for $d$ in the overall formula.

Remark 3.3. Strictly speaking, Theorem 3.2 gives just a safe choice of $d$, and the optimal value might be larger than estimated. For example, let us consider

$$
u(t)-\sqrt{1+(2 t-1)^{2}} \int_{0}^{t} u(s) \mathrm{d} s=t^{2}-\frac{t^{3}}{3} \sqrt{1+(2 t-1)^{2}}, \quad 0 \leq t \leq 1 .
$$

The functions $g$ and $k(\cdot, s)$ in this equation are not analytic at the points $z=$ $(1 \pm \mathrm{i}) / 2$, and the parameter $d$ can be taken as $d=\pi / 2$ in the SE case at most, and as $d=\pi / 6$ in the DE case. However, the solution is $u(t)=t^{2}$, which is analytic on the whole complex plane. Thus, in several exceptional cases, the singularities of $g$ and $k$ might cancel, and the estimated value of $d$ can be too moderate, i.e., smaller than the optimal value. 
3.4. Main result 2: Rigorous proof of the exponential convergence. In Theorem 3.1, there remains the $N$-dependent term $\left\|\left(I_{m}-V_{m}^{\mathrm{DE}}\right)^{-1}\right\|_{2}$. Muhammad et al. 14 numerically confirmed that this term remains low in all of their experiments, which suggests that their scheme converges exponentially. However, the boundedness of this term has not been proven theoretically thus far. Furthermore, in this theorem, the existence of the inverse matrix $\left(I_{m}-V_{m}^{\mathrm{DE}}\right)^{-1}$, which is related to the feasibility of the schemes, is implicitly assumed and not proved.

In the present paper, we rigorously prove the exponential convergence, providing the missing discussions described above. The proof is given in Section 6 .

Theorem 3.4. Let the assumptions in Theorem 3.2 be satisfied with $\mathscr{D}=\psi^{\mathrm{SE}}\left(\mathscr{D}_{d}\right)$. Let $g \in C([a, b])$, and let $k(t, \cdot) Q(\cdot) \in C([a, b])$ and $k(\cdot, s) Q(s) \in C([a, b])$ for all $t, s \in[a, b]$. Then, there exists a positive integer $N_{0}$ such that for all $N \geq N_{0}$, the coefficient matrix $\left(I_{m}-V_{m}^{\mathrm{SE}}\right)$ is invertible. Furthermore, there exists a constant $C$ for all $N \geq N_{0}$ such that

$$
\max _{a \leq t \leq b}\left|u(t)-u_{N}^{\mathrm{SE}}(t)\right| \leq C \mathrm{e}^{-\sqrt{\pi d \alpha N}} .
$$

Theorem 3.5. Let the assumptions in Theorem 3.2 be satisfied with $\mathscr{D}=\psi^{\mathrm{DE}}\left(\mathscr{D}_{d}\right)$. Let $g \in C([a, b])$, and let $k(t, \cdot) Q(\cdot) \in C([a, b])$ and $k(\cdot, s) Q(s) \in C([a, b])$ for all $t, s \in[a, b]$. Then, there exists a positive integer $N_{0}$ such that for all $N \geq N_{0}$, the coefficient matrix $\left(I_{m}-V_{m}^{\mathrm{DE}}\right)$ is invertible. Furthermore, there exists a constant $C$ for all $N \geq N_{0}$ such that

$$
\max _{a \leq t \leq b}\left|u(t)-u_{N}^{\mathrm{DE}}(t)\right| \leq C \frac{\log (2 d N / \alpha)}{N} \exp \left[\frac{-\pi d N}{\log (2 d N / \alpha)}\right] .
$$

Remark 3.6. The assumption that $g$ and $k Q$ belong to $C([a, b])$ is only needed to guarantee the continuity at the endpoints $t=a, b$ since the domains $\psi^{\mathrm{SE}}\left(\mathscr{D}_{d}\right)$ and $\psi^{\mathrm{DE}}\left(\mathscr{D}_{d}\right)$ do not include the endpoints.

3.5. Main result 3: Analysis of conditions of the resulting systems. In Section 3.4, we showed the invertibility of the coefficient matrices appearing in the schemes. In addition, we show stronger results: we prove the boundedness of the condition numbers of the coefficient matrices as follows. The proof is given in Section 7

Theorem 3.7. Let the assumptions in Theorem 3.4 be satisfied. Then, there exists a constant $C_{1}$ independent of $N$ such that

$$
\left\|\left(I_{m}-V_{m}^{\mathrm{SE}}\right)\right\|_{\infty} \leq C_{1} .
$$

Furthermore, there exists a constant $C_{2}$ independent of $N$ such that for all $N \geq N_{0}$,

$$
\left\|\left(I_{m}-V_{m}^{\mathrm{SE}}\right)^{-1}\right\|_{\infty} \leq C_{2},
$$

where $N_{0}$ is the same integer as in Theorem 3.4 .

Theorem 3.8. Let the assumptions in Theorem 3.5 be satisfied. Then, there exists a constant $C_{1}$ independent of $N$ such that

$$
\left\|\left(I_{m}-V_{m}^{\mathrm{DE}}\right)\right\|_{\infty} \leq C_{1} .
$$

Furthermore, there exists a constant $C_{2}$ independent of $N$ such that for all $N \geq N_{0}$,

$$
\left\|\left(I_{m}-V_{m}^{\mathrm{DE}}\right)^{-1}\right\|_{\infty} \leq C_{2}
$$

where $N_{0}$ is the same integer as in Theorem 3.5. 
Remark 3.9. The matrix norm used in the two theorems above is the infinity-norm $\|\cdot\|_{\infty}$, whereas the 2-norm is used in Theorem 3.1. This is due to a technical reason: the norm $\|\cdot\|_{C([a, b])}$ is closely-linked to $\|\cdot\|_{\infty}$.

\section{Numerical Examples}

The main purpose of the numerical experiments described in this section is to investigate the variation in the performance of the Sinc-Nyström methods with the tuning parameter $d$ (related to Section 3.3). The rate of convergence is also confirmed (related to Section 3.4). In addition, we confirm that the resulting system is well conditioned by investigating the condition number (related to Section 3.5).

We used $\mathrm{C}++$ with double precision arithmetic for implementation. GNU Scientific Library (GSL) was used for computing the sine integral function $\operatorname{Si}(x)$, appearing in the basis function $J(j, h)$ defined by (2.2).

4.1. Estimating the tuning parameter $d$. Throughout this section, we consider the following test equation (taken from the handbook [18, Eq. 2.1.50]):

$$
u(t)=\frac{\sqrt{t}}{2}\left\{\frac{6}{1+3 t^{2}}+\log \left(1+3 t^{2}\right)\right\}-\int_{0}^{t} \sqrt{t s} u(s) \mathrm{d} s, \quad 0 \leq t \leq 1 .
$$

Let us first estimate parameter $d$ of the solution $u$ by using Theorem 3.2 , without using any information about the solution. Let $\pi_{\epsilon}=\pi-\epsilon$, where $\epsilon$ denotes an arbitrary small positive number. The functions $g$ and $k$ of (4.1) satisfy the assumptions in Theorem 3.2 with $\mathscr{D}=\psi^{\mathrm{SE}}\left(\mathscr{D}_{2 \pi_{\epsilon} / 3}\right)$ and $\alpha=1$ in the SE case. In the DE case, let us set the following values:

$$
\begin{aligned}
p & =\frac{\pi}{3 \log 2} \\
x & =-\frac{\log 2}{\pi}\left[1-\sqrt{\frac{1+5 p^{2}+\sqrt{\left(1+5 p^{2}\right)^{2}+(4 p)^{2}}}{2}}\right], \\
y & =\frac{2}{3}\left[1-\sqrt{\frac{2}{1+5 p^{2}+\sqrt{\left(1+5 p^{2}\right)^{2}+(4 p)^{2}}}}\right] \\
d_{\epsilon} & =\arcsin \left(\frac{y}{\sqrt{x^{2}+y^{2}}}\right)-\epsilon .
\end{aligned}
$$

Then, the assumptions in Theorem 3.2 are fulfilled with $\mathscr{D}=\psi^{\mathrm{DE}}\left(\mathscr{D}_{d_{\epsilon}}\right)$ and $\alpha=1$. Hence, parameter $d$ can be estimated as $d=2 \pi_{\epsilon} / 3 \simeq 2.09$ in the SE case and $d=$ $d_{\epsilon} \simeq 0.69$ in the DE case. The analytical solution of (4.1) is $u(t)=3 \sqrt{t} /\left(1+3 t^{2}\right)$, which actually belongs to $\mathbf{H}^{\infty}\left(\psi^{\mathrm{SE}}\left(\mathscr{D}_{2 \pi_{\epsilon} / 3}\right)\right)$ and $\mathbf{H}^{\infty}\left(\psi^{\mathrm{DE}}\left(\mathscr{D}_{d_{\epsilon}}\right)\right)$. Thus, parameter $d$ can be estimated by Theorem 3.2 without the solution.

4.2. Variation in performance with parameter $d$. The parameter $d$ estimated above is used for computing the mesh size $h$, defined by (2.5) or (2.6). We are interested in the variation in the performance of the schemes when $d$ is selected incorrectly. Therefore, we define $h$ as

$$
h=\sqrt{\frac{2^{r} \pi_{\epsilon} \pi}{3 N}}
$$

in the SE case and

$$
h=\frac{\log \left(2^{r} d_{\epsilon} N\right)}{N}
$$


in the DE case, and we conduct numerical experiments with $r=-1,0,1,2,3$ $(r=1$ is optimal in theory). The results are shown in Figures 1 4. We checked the error of the numerical solutions $u_{N}^{\mathrm{SE}}$ and $u_{N}^{\mathrm{DE}}$ on 101 equally-spaced points over the interval $[0,1]$, and the maximum errors among them are shown in these graphs. We observe that in the SE case (Figures 1 and 21), the performance is remarkably degraded as $|r-1|$ increases. The convergence rate in all cases is consistently observed as $\mathrm{O}\left(\exp \left(-c_{1} \sqrt{N}\right)\right)$, although $c_{1}$ is different in each case. In the DE case (Figures 3 and 4), the convergence rate in all cases is consistently observed as $\mathrm{O}\left(\exp \left(-c_{2} N / \log N\right)\right)$, but the difference in the results is interesting. Although there is no significant difference as in the SE case, the convergence rate appears to improve as $r$ decreases. Discussions on these observations are given from a theoretical viewpoint in Section 4.4



Figure 1. Performance of the SE-Sinc-Nyström method with $h$ defined by (4.2), $r=-1,0,1$.

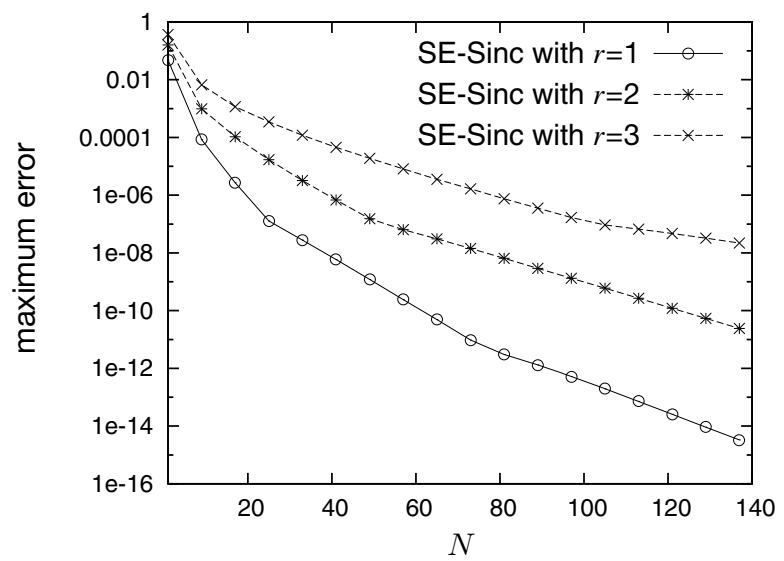

Figure 2. Performance of the SE-Sinc-Nyström method with $h$ defined by (4.2), $r=1,2,3$. 


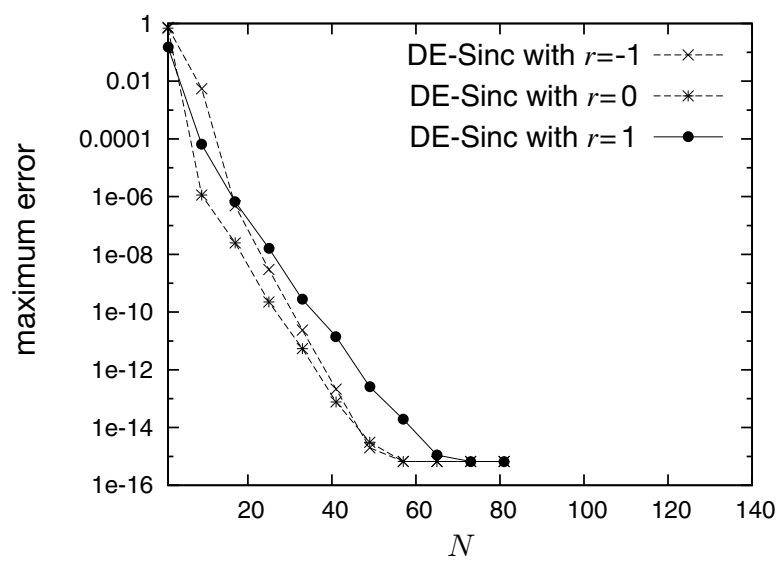

Figure 3. Performance of the DE-Sinc-Nyström method with $h$ defined by (4.3), $r=-1,0,1$.

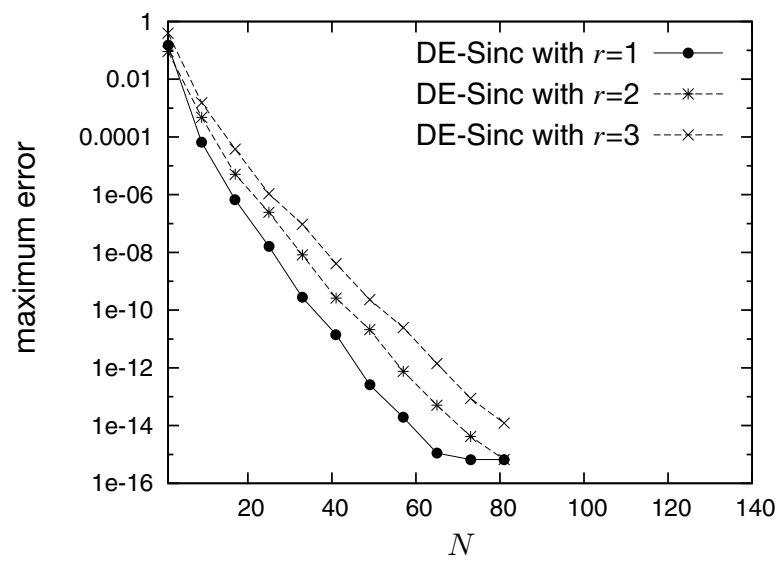

Figure 4. Performance of the DE-Sinc-Nyström method with $h$ defined by (4.3), $r=1,2,3$.

4.3. Checking the condition numbers of the coefficient matrices. We also investigate the condition numbers

$$
\left\|\left(I_{m}-V_{m}^{\mathrm{SE}}\right)\right\|_{\infty}\left\|\left(I_{m}-V_{m}^{\mathrm{SE}}\right)^{-1}\right\|_{\infty} \quad \text { and } \quad\left\|\left(I_{m}-V_{m}^{\mathrm{DE}}\right)\right\|_{\infty}\left\|\left(I_{m}-V_{m}^{\mathrm{DE}}\right)^{-1}\right\|_{\infty}
$$

in the case of $r=1$. The results are shown in Figure 5] from which we can see that the condition numbers remain quite low and bounded, at least in this range of $N$.

4.4. Discussions on the observed convergence rates. Let us roughly explain the convergence rates observed in Section 4.2 by analyzing the (SE/DE) Sinc indefinite integration, since it determines the performance of the Sinc-Nyström methods as shown in Section 6. The SE-Sinc indefinite integration has two main error terms, the "discretization error" and the "truncation error", which can be estimated as 


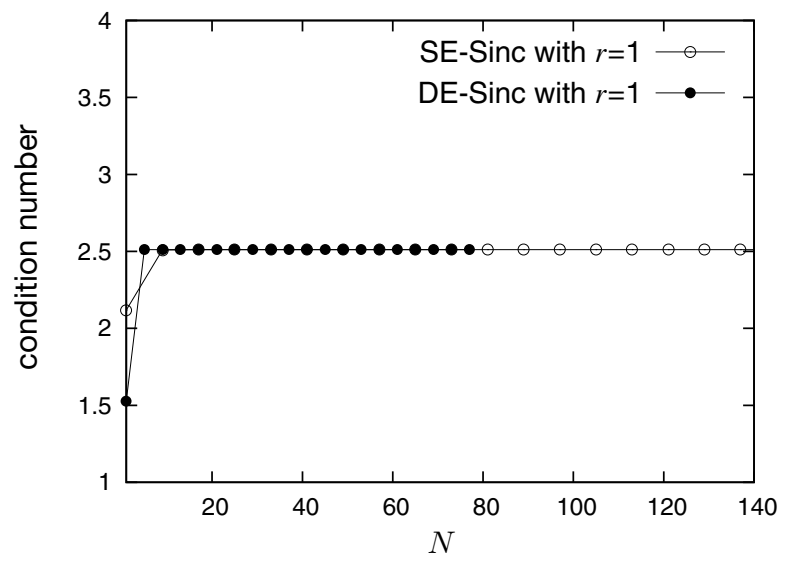

Figure 5. Condition number in the SE- and DE-Sinc-Nyström methods.

$\mathrm{O}\left(h \mathrm{e}^{-\pi d / h}\right)$ and $\mathrm{O}\left(\mathrm{e}^{-\alpha N h}\right)$ [21, respectively. Then, we can estimate the whole error $E_{\mathrm{SE}-\text { Sinc }}$ as

$$
E_{\text {SE-Sinc }} \leq C_{\mathrm{D}} h \mathrm{e}^{-\pi d / h}+C_{\mathrm{T}} \mathrm{e}^{-\alpha N h} .
$$

If we define the mesh size $h$ as

$$
h=\sqrt{\frac{c}{N}}
$$

(recall (4.2), where $c=2^{r-1} \pi d / \alpha$, and where $d=2 \pi_{\epsilon} / 3$ and $\alpha=1$ ), we have

$$
E_{\text {SE-Sinc }} \leq C_{\mathrm{D}} \sqrt{\frac{c}{N}} \mathrm{e}^{-\pi d \sqrt{N / c}}+C_{\mathrm{T}} \mathrm{e}^{-\alpha \sqrt{c N}},
$$

and we easily see that $c=\pi d / \alpha$ is optimal, giving the rate $\mathrm{O}\left(\mathrm{e}^{-\sqrt{\pi d \alpha N}}\right)$. If $c$ exceeds this value, the first term limits the convergence rate to $\mathrm{O}\left(\mathrm{e}^{-\pi d \sqrt{N / c}} / \sqrt{N}\right)$. Similarly, if $c$ is smaller than this value, the rate becomes $\mathrm{O}\left(\mathrm{e}^{-\alpha \sqrt{c N}}\right)$. Thus, we can conclude that in all cases, the convergence rate is consistently $\mathrm{O}\left(\exp \left(-c_{1} \sqrt{N}\right)\right)$, as observed in the numerical experiments.

Next, we consider the DE case. The DE-Sinc indefinite integration also has the two main error terms stated above, but the truncation error is different from the SE case: $\mathrm{O}\left(\mathrm{e}^{-\frac{\pi}{2} \exp (N h)}\right)$ [17]. Therefore, the whole error $E_{\mathrm{DE}-\mathrm{Sinc}}$ can be estimated as

$$
E_{\text {DE-Sinc }} \leq C_{\mathrm{D}} h \mathrm{e}^{-\pi d / h}+C_{\mathrm{T}} \mathrm{e}^{-\frac{\pi}{2} \exp (N h)} .
$$

If we define the mesh size $h$ as

$$
h=\frac{\log (c N)}{N}
$$

(recall (4.3), where $c=2^{r} d / \alpha$, and where $d=d_{\epsilon}$ and $\alpha=1$ ), we have

$$
\begin{aligned}
E_{\mathrm{DE}-\mathrm{Sinc}} & \leq C_{\mathrm{D}} \frac{\log (c N)}{N} \mathrm{e}^{-\pi d N / \log (c N)}+C_{\mathrm{T}} \mathrm{e}^{-\frac{\pi}{2} c N} \\
& =\left\{C_{\mathrm{D}}+C_{\mathrm{T}} \frac{N}{\log (c N)} \mathrm{e}^{-\frac{\pi}{2} N(c-2 d / \log (c N))}\right\} \frac{\log (c N)}{N} \mathrm{e}^{-\pi d N / \log (c N)} .
\end{aligned}
$$


Since the part in $\{\cdot\}$ can be bounded by a constant $\tilde{C}$ independent of $N$, the convergence rate is $\mathrm{O}\left(\log (c N) \mathrm{e}^{-\pi d N / \log (c N)} / N\right)$. From the estimate, we see that the rate can be improved by taking $c$ as small as possible, as roughly observed in the numerical experiments.

However, we should note that if $c$ is taken as too small, the part in $\{\cdot\}$ becomes large. Actually, in the small range of $N$ in Figure 3, the error becomes larger as $r$ decreases, which suggests that the constant $\tilde{C}$ becomes larger. On the other hand, in Figure 4 the error when $r=1$ (the standard case) is smaller than the others over almost the entire range of $N$. Considering the numerical experiments and the theoretical estimates stated above, we can say that the standard formula (2.6) ( $r=1$ in the experiments) represents a well-balanced way to select $h$ from the viewpoints of the convergence rate and the constant.

\section{Proof of MAin Result 1}

The idea here is to apply the standard contraction mapping theorem. Let $\mathscr{D}=$ $\psi^{\mathrm{SE}}\left(\mathscr{D}_{d}\right)$ or $\mathscr{D}=\psi^{\mathrm{DE}}\left(\mathscr{D}_{d}\right)$, and let us introduce integral operators $\mathcal{V}: \mathbf{H}^{\infty}(\mathscr{D}) \rightarrow$ $\mathbf{H}^{\infty}(\mathscr{D})$ as

$$
\mathcal{V}[f](t)=\int_{a}^{t} k(t, s) f(s) \mathrm{d} s,
$$

where the kernel $k$ is assumed to satisfy the assumptions in Theorem 3.2. This operator becomes a contraction map by repeated multiplication, as stated below.

Lemma 5.1. Let $\mathscr{D}=\psi^{\mathrm{SE}}\left(\mathscr{D}_{d}\right)$ or $\mathscr{D}=\psi^{\mathrm{DE}}\left(\mathscr{D}_{d}\right)$. Let $k(z, \cdot) Q(\cdot) \in \mathbf{L}_{\alpha}(\mathscr{D})$ and $k(\cdot, w) Q(w) \in \mathbf{H}^{\infty}(\mathscr{D})$ for all $z, w \in \mathscr{D}$. Furthermore, let $f \in \mathbf{H}^{\infty}(\mathscr{D})$. Then, it holds for all positive integers $n$ that

$$
\left\|\mathcal{V}^{n} f\right\|_{\mathbf{H}^{\infty}(\mathscr{D})} \leq \frac{\left\{K(b-a)^{2 \alpha-1} c_{\alpha, d}\right\}^{n}}{n !}\|f\|_{\mathbf{H}^{\infty}(\mathscr{D})},
$$

where $K$ is a constant depending only on $k$, and $c_{\alpha, d}$ is a constant depending only on $\alpha$ and $d$.

From this lemma, (1.1), which can be rewritten symbolically as $(\mathcal{I}-\mathcal{V}) u=g$, has a unique solution $u \in \mathbf{H}^{\infty}(\mathscr{D})$ by the contraction mapping theorem (note that $\mathbf{H}^{\infty}(\mathscr{D})$ is a Banach space [20, $\left.\left.\S 11.31\right]\right)$. Thus, Theorem 3.2 is established.

In what follows, we prove Lemma 5.1 considering the SE and DE cases separately. The next lemma is needed for the SE case.

Lemma 5.2 (Okayama et al. [15, Lemma A.1]). Let $d$ be a constant with $0<d<\pi$ and let us define a function $\psi_{1}$ as

$$
\psi_{1}(x)=\frac{1}{2} \tanh \left(\frac{x}{2}\right)+\frac{1}{2} .
$$

Then, there exists a constant $c_{1}$ depending only on $d$, such that for all $x \in \mathbb{R}$ and $y \in[-d, d]$,

$$
\left|Q\left(\psi^{\mathrm{SE}}(x+\mathrm{i} y)\right)\right| \leq(b-a)^{2} c_{1} \psi_{1}^{\prime}(x) .
$$

The following lemma is sufficient to establish Lemma 5.1 with $\mathscr{D}=\psi^{\mathrm{SE}}\left(\mathscr{D}_{d}\right)$.

Lemma 5.3. Let the assumptions in Lemma 5.1 be satisfied with $\mathscr{D}=\psi^{\mathrm{SE}}\left(\mathscr{D}_{d}\right)$. Then, it holds for all positive integers $n$ and $z \in \mathscr{D}$ that

$$
\left|\mathcal{V}^{n}[f](z)\right| \leq \frac{\left\{K(b-a)^{2 \alpha-1} c_{1}^{\alpha} \mathrm{B}\left(\psi_{1}(x), \alpha, \alpha\right)\right\}^{n}}{n !}\|f\|_{\mathbf{H}^{\infty}(\mathscr{D})},
$$


where $x=\operatorname{Re}\left[\left\{\psi^{\mathrm{SE}}\right\}^{-1}(z)\right], \mathrm{B}(t, \alpha, \beta)$ is the incomplete beta function, $K$ is the constant in (2.3) regarding $k(z, \cdot) Q(\cdot) \in \mathbf{L}_{\alpha}(\mathscr{D})$, and $c_{1}$ is a constant depending only on $d$.

Proof. Let $y=\operatorname{Im}\left[\left\{\psi^{\mathrm{SE}}\right\}^{-1}(z)\right]$, and accordingly, $z=\psi^{\mathrm{SE}}(x+\mathrm{i} y)$. Let $\gamma=K(b-$ $a)^{2 \alpha-1} c_{1}^{\alpha}$ for short. We show this lemma by induction. First, consider the case $n=1$. By the variable transformation $w=\psi^{\mathrm{SE}}(t+\mathrm{i} y)$, we have

$$
\begin{aligned}
\mathcal{V}[f](z) & =\int_{a}^{z} k(z, w) f(w) \mathrm{d} w \\
& =\int_{-\infty}^{x} k\left(z, \psi^{\mathrm{SE}}(t+\mathrm{i} y)\right) f\left(\psi^{\mathrm{SE}}(t+\mathrm{i} y)\right)\left\{\psi^{\mathrm{SE}}\right\}^{\prime}(t+\mathrm{i} y) \mathrm{d} t \\
& =\int_{-\infty}^{x} k\left(z, \psi^{\mathrm{SE}}(t+\mathrm{i} y)\right) f\left(\psi^{\mathrm{SE}}(t+\mathrm{i} y)\right) \frac{Q\left(\psi^{\mathrm{SE}}(t+\mathrm{i} y)\right)}{b-a} \mathrm{~d} t .
\end{aligned}
$$

From $k(z, \cdot) Q(\cdot) \in \mathbf{L}_{\alpha}(\mathscr{D})$ and Lemma [5.2, it holds that

$$
\begin{aligned}
|\mathcal{V}[f](z)| & \leq \frac{\|f\|_{\mathbf{H}^{\infty}(\mathscr{D})} K}{b-a} \int_{-\infty}^{x}\left|Q\left(\psi^{\mathrm{SE}}(t+\mathrm{i} y)\right)\right|^{\alpha} \mathrm{d} t \\
& \leq \frac{\|f\|_{\mathbf{H}^{\infty}(\mathscr{D})} K}{b-a} \int_{-\infty}^{x}\left\{(b-a)^{2} c_{1} \psi_{1}^{\prime}(t)\right\}^{\alpha} \mathrm{d} t \\
& =\|f\|_{\mathbf{H}^{\infty}(\mathscr{D})} \gamma \mathrm{B}\left(\psi_{1}(x), \alpha, \alpha\right) .
\end{aligned}
$$

Hence, (5.1) holds when $n=1$ (recall $\left.\gamma=K(b-a)^{2 \alpha-1} c_{1}^{\alpha}\right)$.

Next, assume that (5.1) holds at $n$ and consider $n+1$. It holds that

$$
\begin{aligned}
\left|\mathcal{V}\left[\mathcal{V}^{n} f\right](z)\right| & =\left|\int_{-\infty}^{x} k\left(z, \psi^{\mathrm{SE}}(t+\mathrm{i} y)\right) \mathcal{V}^{n}[f]\left(\psi^{\mathrm{SE}}(t+\mathrm{i} y)\right) \frac{Q\left(\psi^{\mathrm{SE}}(t+\mathrm{i} y)\right)}{b-a} \mathrm{~d} t\right| \\
& \leq \int_{-\infty}^{x} \frac{K\left|Q\left(\psi^{\mathrm{SE}}(t+\mathrm{i} y)\right)\right|^{\alpha}}{b-a} \frac{\left\{\gamma \mathrm{B}\left(\psi_{1}(t), \alpha, \alpha\right)\right\}^{n}}{n !}\|f\|_{\mathbf{H}^{\infty}(\mathscr{D})} \mathrm{d} t \\
& \leq\|f\|_{\mathbf{H}^{\infty}(\mathscr{D})} \gamma^{n+1} \int_{-\infty}^{x}\left\{\psi_{1}^{\prime}(t)\right\}^{\alpha} \frac{\left\{\mathrm{B}\left(\psi_{1}(t), \alpha, \alpha\right)\right\}^{n}}{n !} \mathrm{d} t \\
& =\|f\|_{\mathbf{H}^{\infty}(\mathscr{D})} \gamma^{n+1} \frac{\left\{\mathrm{B}\left(\psi_{1}(x), \alpha, \alpha\right)\right\}^{n+1}}{(n+1) !} .
\end{aligned}
$$

This completes the proof.

Next, let us consider the DE case. For this purpose, the next lemma is needed.

Lemma 5.4 (Okayama et al. [15, Lemma A.4]). Let $d$ be a constant with $0<d<$ $\pi / 2$, and let us define a function $\psi_{2}$ as

$$
\psi_{2}(x)=\frac{1}{2} \tanh \left(\frac{\pi \cos d}{2} \sinh (x)\right)+\frac{1}{2} .
$$

Then, there exists a constant $c_{2}$ depending only on $d$, such that for all $x \in \mathbb{R}$ and $y \in[-d, d]$,

$$
\left|\pi \cosh (x+\mathrm{i} y) Q\left(\psi^{\mathrm{DE}}(x+\mathrm{i} y)\right)\right| \leq(b-a)^{2} c_{2} \psi_{2}^{\prime}(x) .
$$

The following lemma is sufficient to establish Lemma 5.1 with $\mathscr{D}=\psi^{\mathrm{DE}}\left(\mathscr{D}_{d}\right)$. 
Lemma 5.5. Let the assumptions in Lemma 5.1 be satisfied with $\mathscr{D}=\psi^{\mathrm{DE}}\left(\mathscr{D}_{d}\right)$. Then, it holds for all positive integers $n$ and $z \in \mathscr{D}$ that

$$
\left|\mathcal{V}^{n}[f](z)\right| \leq \frac{\left\{K(b-a)^{2 \alpha-1} c_{2}^{\prime} \mathrm{B}\left(\psi_{2}(x), \alpha, \alpha\right)\right\}^{n}}{n !}\|f\|_{\mathbf{H}^{\infty}(\mathscr{D})},
$$

where $x=\operatorname{Re}\left[\left\{\psi^{\mathrm{DE}}\right\}^{-1}(z)\right], K$ is the constant in (2.3) regarding $k(z, \cdot) Q(\cdot) \in$ $\mathbf{L}_{\alpha}(\mathscr{D})$, and $c_{2}^{\prime}=\left(c_{2}^{\alpha} / \cos ^{1-\alpha} d\right)$, where $c_{2}$ is a constant depending only on $d$.

Proof. Let $y=\operatorname{Im}\left[\left\{\psi^{\mathrm{DE}}\right\}^{-1}(z)\right]$, and accordingly, $z=\psi^{\mathrm{DE}}(x+\mathrm{i} y)$. Let $\gamma=$ $K(b-a)^{2 \alpha-1} c_{2}^{\prime}$ for short. We show this lemma by induction. First, consider the case $n=1$. By the variable transformation $w=\psi^{\mathrm{DE}}(t+\mathrm{i} y)$, we have

$$
\begin{aligned}
\mathcal{V}[f](z) & =\int_{a}^{z} k(z, w) f(w) \mathrm{d} w \\
& =\int_{-\infty}^{x} k\left(z, \psi^{\mathrm{DE}}(t+\mathrm{i} y)\right) f\left(\psi^{\mathrm{DE}}(t+\mathrm{i} y)\right)\left\{\psi^{\mathrm{DE}}\right\}^{\prime}(t+\mathrm{i} y) \mathrm{d} t \\
& =\int_{-\infty}^{x} k\left(z, \psi^{\mathrm{DE}}(t+\mathrm{i} y)\right) f\left(\psi^{\mathrm{DE}}(t+\mathrm{i} y)\right) \frac{\pi \cosh (t+\mathrm{i} y) Q\left(\psi^{\mathrm{DE}}(t+\mathrm{i} y)\right)}{b-a} \mathrm{~d} t .
\end{aligned}
$$

From $k(z, \cdot) Q(\cdot) \in \mathbf{L}_{\alpha}(\mathscr{D})$ and Lemma 5.4, it holds that

$$
\begin{aligned}
|\mathcal{V}[f](z)| & \leq \frac{\|f\|_{\mathbf{H}^{\infty}(\mathscr{D})} K}{b-a} \int_{-\infty}^{x} \pi|\cosh (t+\mathrm{i} y)| \cdot\left|Q\left(\psi^{\mathrm{DE}}(t+\mathrm{i} y)\right)\right|^{\alpha} \mathrm{d} t \\
& \leq \frac{\|f\|_{\mathbf{H}^{\infty}(\mathscr{D})} K}{b-a} \int_{-\infty}^{x}\{\pi \cosh (t)\}^{1-\alpha}\left\{(b-a)^{2} c_{2} \psi_{2}^{\prime}(t)\right\}^{\alpha} \mathrm{d} t \\
& =\|f\|_{\mathbf{H}^{\infty}(\mathscr{D})} \gamma \mathrm{B}\left(\psi_{2}(x), \alpha, \alpha\right) .
\end{aligned}
$$

Hence, (5.2) holds when $n=1$ (recall $\gamma=K(b-a)^{2 \alpha-1} c_{2}^{\prime}$ ).

Next, assume that (5.2) holds at $n$ and consider $n+1$. It holds that

$$
\begin{aligned}
& \left|\mathcal{V}\left[\mathcal{V}^{n} f\right](z)\right| \\
& =\left|\int_{-\infty}^{x} k\left(z, \psi^{\mathrm{DE}}(t+\mathrm{i} y)\right) \mathcal{V}^{n}[f]\left(\psi^{\mathrm{DE}}(t+\mathrm{i} y)\right) \frac{\pi \cosh (t+\mathrm{i} y) Q\left(\psi^{\mathrm{DE}}(t+\mathrm{i} y)\right)}{b-a} \mathrm{~d} t\right| \\
& \leq \int_{-\infty}^{x} \frac{\pi|\cosh (t+\mathrm{i} y)| K\left|Q\left(\psi^{\mathrm{DE}}(t+\mathrm{i} y)\right)\right|^{\alpha}}{b-a} \frac{\left\{\gamma \mathrm{B}\left(\psi_{2}(t), \alpha, \alpha\right)\right\}^{n}}{n !}\|f\|_{\mathbf{H}^{\infty}(\mathscr{D})} \mathrm{d} t \\
& \leq\|f\|_{\mathbf{H}^{\infty}(\mathscr{D})} \gamma^{n+1} \int_{-\infty}^{x}\{\pi \cosh (t) \cos d\}^{1-\alpha}\left\{\psi_{2}^{\prime}(t)\right\}^{\alpha} \frac{\left\{\mathrm{B}\left(\psi_{2}(t), \alpha, \alpha\right)\right\}^{n}}{n !} \mathrm{d} t \\
& =\|f\|_{\mathbf{H}^{\infty}(\mathscr{D})} \gamma^{n+1} \frac{\left\{\mathrm{B}\left(\psi_{2}(x), \alpha, \alpha\right)\right\}^{n+1}}{(n+1) !} .
\end{aligned}
$$

This completes the proof.

\section{Proof of main Result 2}

In this section, we prove Theorems 3.4 and 3.5. Note that $\|\cdot\|_{C([a, b])}$ is used as the norm in this section, although $\|\cdot\|_{\mathbf{H}^{\infty}(\mathscr{D})}$ is used in the previous section. This is because we should evaluate the error not on the complex domain $\mathscr{D}$, but on the interval $[a, b]$. We write $\mathbf{C}=C([a, b])$ for short, and the operators appearing in this section, such as $\mathcal{V}, \mathcal{V}_{N}^{\mathrm{SE}}$, and $\mathcal{V}_{N}^{\mathrm{DE}}$, are assumed to map $\mathbf{C}$ onto $\mathbf{C}$.

6.1. Convergence analysis of the SE-Sinc-Nyström methods. First, we prove the SE case, i.e., Theorem 3.4 
6.1.1. Sketch of the proof. Let us define an operator $\mathcal{V}_{N}^{\mathrm{SE}}$ as

$$
\mathcal{V}_{N}^{\mathrm{SE}}[f](t)=\sum_{j=-N}^{N} k\left(t, t_{j}^{\mathrm{SE}}\right) f\left(t_{j}^{\mathrm{SE}}\right)\left\{\psi^{\mathrm{SE}}\right\}^{\prime}(j h) J(j, h)\left(\left\{\psi^{\mathrm{SE}}\right\}^{-1}(t)\right),
$$

which is the approximation of $\mathcal{V} f$ by the SE-Sinc indefinite integration. Then, consider the following three equations:

$$
\begin{aligned}
(\mathcal{I}-\mathcal{V}) u & =g, \\
\left(\mathcal{I}-\mathcal{V}_{N}^{\mathrm{SE}}\right) v & =g, \\
\left(I_{m}-V_{m}^{\mathrm{SE}}\right) \boldsymbol{c}_{m} & =\boldsymbol{g}_{m}^{\mathrm{SE}} .
\end{aligned}
$$

The first equation is nothing but (1.1), the second is (3.1), and the third is (3.4). In order to prove the existence of $\left(I_{m}-V_{m}^{\mathrm{SE}}\right)^{-1}$, which represents the feasibility of the scheme, we prove the following two claims:

(1) Equation (6.2) is uniquely solvable if and only if (6.1) is uniquely solvable (Lemma 6.1);

(2) Equation (6.1) is uniquely solvable for all sufficiently large $N$ (Lemma 6.7). Combining Lemmas 6.1 and 6.7, we conclude Theorem 3.4.

\subsubsection{Step 1: Equivalence of the solvability of (6.2) and (6.1).}

Lemma 6.1. The following statements are equivalent:

(A) Equation (6.1) has a unique solution $v \in \mathbf{C}$.

(B) Equation (6.2) has a unique solution $\boldsymbol{c}_{m} \in \mathbb{R}^{m}$.

Proof. First, we prove that $(\mathrm{A}) \Rightarrow(\mathrm{B})$. If we define $\boldsymbol{c}_{m}$ as $\boldsymbol{c}_{m}=\left[v\left(t_{-N}^{\mathrm{SE}}\right), \ldots, v\left(t_{N}^{\mathrm{SE}}\right)\right]^{\mathrm{T}}$, clearly, $\boldsymbol{c}_{m}$ is a solution of (6.2). Next, we prove the uniqueness. Let $\tilde{\boldsymbol{c}}_{m}=$ $\left[\tilde{c}_{-N}, \ldots, \tilde{c}_{N}\right]^{\mathrm{T}}$ be another solution of (6.2). Then, let us define a function $\tilde{v} \in \mathbf{C}$ with the vector as

$$
\tilde{v}(t)=g(t)+\sum_{j=-N}^{N} k\left(t, t_{j}^{\mathrm{SE}}\right) \tilde{c}_{j}\left\{\psi^{\mathrm{SE}}\right\}^{\prime}(j h) J(j, h)\left(\left\{\psi^{\mathrm{SE}}\right\}^{-1}(t)\right) .
$$

On the SE-Sinc points, $t=t_{i}^{\mathrm{SE}}(i=-N, \ldots, N)$, it holds that

$$
\tilde{v}\left(t_{i}^{\mathrm{SE}}\right)=g\left(t_{i}^{\mathrm{SE}}\right)+\sum_{j=-N}^{N} k\left(t_{i}^{\mathrm{SE}}, t_{j}^{\mathrm{SE}}\right) \tilde{c}_{j}\left\{\psi^{\mathrm{SE}}\right\}^{\prime}(j h) J(j, h)(i h) .
$$

Furthermore, since $\tilde{\boldsymbol{c}}$ is a solution of (6.2), it holds that

$$
\tilde{c}_{i}=g\left(t_{i}^{\mathrm{SE}}\right)+\sum_{j=-N}^{N} k\left(t_{i}^{\mathrm{SE}}, t_{j}^{\mathrm{SE}}\right) \tilde{c}_{j}\left\{\psi^{\mathrm{SE}}\right\}^{\prime}(j h) J(j, h)(i h) .
$$

Therefore, $\tilde{v}\left(t_{i}^{\mathrm{SE}}\right)=\tilde{c}_{i}$, and from this, (6.3) can be rewritten as $\left(\mathcal{I}-\mathcal{V}_{N}^{\mathrm{SE}}\right) \tilde{v}=g$, which shows that $\tilde{v}$ is a solution of (6.1). By assumption (A), $v=\tilde{v}$ holds, and thus, $\boldsymbol{c}_{m}=\tilde{\boldsymbol{c}}_{m}$. This shows the desired uniqueness.

Next, we prove that $(\mathrm{B}) \Rightarrow(\mathrm{A})$. Let us define a function $v \in \mathbf{C}$ as

$$
v(t)=g(t)+\sum_{j=-N}^{N} k\left(t, t_{j}^{\mathrm{SE}}\right) c_{j}\left\{\psi^{\mathrm{SE}}\right\}^{\prime}(j h) J(j, h)\left(\left\{\psi^{\mathrm{SE}}\right\}^{-1}(t)\right) .
$$


Then, by the same argument as above, $v\left(t_{i}^{\mathrm{SE}}\right)=c_{i}$ holds, from which we have $\left(\mathcal{I}-\mathcal{V}_{N}^{\mathrm{SE}}\right) v=g$. Therefore, (6.1) has a solution. Next, we prove the uniqueness. Let $\tilde{v}$ be another solution of (6.1). By using $\tilde{v}$, let us define $\tilde{\boldsymbol{c}}_{m}$ as $\tilde{\boldsymbol{c}}_{m}=$ $\left[\tilde{v}\left(t_{-N}^{\mathrm{SE}}\right), \ldots, \tilde{v}\left(t_{N}^{\mathrm{SE}}\right)\right]^{\mathrm{T}}$. Clearly, $\tilde{\boldsymbol{c}}_{m}$ is a solution of (6.2), and by assumption (B), $\boldsymbol{c}_{m}=\tilde{\boldsymbol{c}}_{m}$ holds. Therefore, $\tilde{v}\left(t_{j}^{\mathrm{SE}}\right)=c_{j}$ holds, and from this, the equation $(\mathcal{I}-$ $\left.\mathcal{V}_{N}^{\mathrm{SE}}\right) \tilde{v}=g$ can be rewritten as

$$
\tilde{v}(t)=g(t)+\sum_{j=-N}^{N} k\left(t, t_{j}^{\mathrm{SE}}\right) c_{j}\left\{\psi^{\mathrm{SE}}\right\}^{\prime}(j h) J(j, h)\left(\left\{\psi^{\mathrm{SE}}\right\}^{-1}(t)\right) .
$$

Comparing the right-hand sides of (6.4) and (6.5), we conclude that $v=\tilde{v}$, which shows the desired uniqueness.

6.1.3. Step 2: Solvability of (6.1) for all sufficiently large N. For the analysis of Nyström methods, the next theorem is known, and is crucial for our purpose.

Theorem 6.2 (Atkinson [2, Theorem 4.1.1]). Assume the following four conditions:

1. Operators $\mathcal{X}$ and $\mathcal{X}_{n}$ are bounded operators on $\mathbf{C}$ to $\mathbf{C}$.

2. Operator $(\mathcal{I}-\mathcal{X}): \mathbf{C} \rightarrow \mathbf{C}$ has a bounded inverse $(\mathcal{I}-\mathcal{X})^{-1}: \mathbf{C} \rightarrow \mathbf{C}$.

3. Operator $\mathcal{X}_{n}$ is compact on $\mathbf{C}$.

4. The following inequality holds:

$$
\left\|\left(\mathcal{X}-\mathcal{X}_{n}\right) \mathcal{X}_{n}\right\|_{\mathcal{L}(\mathbf{C}, \mathbf{C})}<\frac{1}{\left\|(\mathcal{I}-\mathcal{X})^{-1}\right\|_{\mathcal{L}(\mathbf{C}, \mathbf{C})}} .
$$

Then, $\left(\mathcal{I}-\mathcal{X}_{n}\right)^{-1}$ exists as a bounded operator on $\mathbf{C}$ to $\mathbf{C}$, with

$$
\left\|\left(\mathcal{I}-\mathcal{X}_{n}\right)^{-1}\right\|_{\mathcal{L}(\mathbf{C}, \mathbf{C})} \leq \frac{1+\left\|(\mathcal{I}-\mathcal{X})^{-1}\right\|_{\mathcal{L}(\mathbf{C}, \mathbf{C})}\left\|\mathcal{X}_{n}\right\|_{\mathcal{L}(\mathbf{C}, \mathbf{C})}}{1-\left\|(\mathcal{I}-\mathcal{X})^{-1}\right\|_{\mathcal{L}(\mathbf{C}, \mathbf{C})}\left\|\left(\mathcal{X}-\mathcal{X}_{n}\right) \mathcal{X}_{n}\right\|_{\mathcal{L}(\mathbf{C}, \mathbf{C})}}
$$

Furthermore, if $(\mathcal{I}-\mathcal{X}) u=g$ and $\left(\mathcal{I}-\mathcal{X}_{n}\right) v=g$,

$$
\|u-v\|_{\mathbf{C}} \leq\left\|\left(\mathcal{I}-\mathcal{X}_{n}\right)^{-1}\right\|_{\mathcal{L}(\mathbf{C}, \mathbf{C})}\left\|\left(\mathcal{X}-\mathcal{X}_{n}\right) u\right\|_{\mathbf{C}} .
$$

In what follows, we show that the four conditions are fulfilled with $\mathcal{X}=\mathcal{V}$ and $\mathcal{X}_{n}=\mathcal{V}_{N}^{\mathrm{SE}}$, under the assumptions in Theorem 3.4. Condition 1 clearly holds. Condition 2 is well known (in fact, we can prove it in the same way as in Section 5 , by using the contraction mapping theorem). Condition 3 immediately follows from the Arzelá-Ascoli theorem. The main difficulty of this project lies in condition 4. For this purpose, we need a bound on the basis function $J(j, h)(x)=\int_{-\infty}^{x} S(j, h)(t) \mathrm{d} t$ as follows.

Lemma 6.3 (Stenger [21, Lemma 3.6.5]). For all $x \in \mathbb{R}$, it holds that

$$
|J(j, h)(x)| \leq 1.1 h .
$$

This result can be extended to the complex plane as follows.

Lemma 6.4. For all $x \in \mathbb{R}$ and $y \in \mathbb{R}$, it holds that

$$
|J(j, h)(x+\mathrm{i} y)| \leq \frac{5 h}{\pi} \cdot \frac{\sinh (\pi y / h)}{\pi y / h} .
$$

Proof. We split the integral path as

$J(j, h)(x+\mathrm{i} y)=\int_{-\infty}^{x+\mathrm{i} y} S(j, h)(\zeta) \mathrm{d} \zeta=\int_{-\infty}^{x} S(j, h)(\xi) \mathrm{d} \xi+\int_{0}^{y} S(j, h)(x+\mathrm{i} \eta) \mathrm{d} \eta$, 
and we evaluate the two terms one by one. From Lemma 6.3, the first term can be bounded as

$$
\left|\int_{-\infty}^{x} S(j, h)(\xi) \mathrm{d} \xi\right| \leq 1.1 h<\frac{7 h}{2 \pi} \leq \frac{7 h}{2 \pi} \cdot \frac{\sinh (\pi y / h)}{\pi y / h} .
$$

Next, we evaluate the second term. Note that the inequality

$$
|S(j, h)(x+\mathrm{i} \eta)|=\frac{h}{2 \pi}\left|\int_{-\pi / h}^{\pi / h} \mathrm{e}^{\eta t+\mathrm{i}(j h-x) t} \mathrm{~d} t\right| \leq \frac{h}{2 \pi} \int_{-\pi / h}^{\pi / h} \mathrm{e}^{\eta t} \mathrm{~d} t=\frac{\sinh (\pi \eta / h)}{\pi \eta / h}
$$

holds; furthermore,

$$
\int_{0}^{|r|} \frac{\sinh t}{t} \mathrm{~d} t \leq \frac{3}{2} \frac{\sinh r}{r}
$$

holds for all $r \in \mathbb{R}$. Then, we have

$$
\begin{aligned}
\left|\int_{0}^{y} S(j, h)(x+\mathrm{i} \eta) \mathrm{d} \eta\right| \leq \int_{0}^{|y|} \frac{\sinh (\pi \eta / h)}{\pi \eta / h} \mathrm{~d} \eta & =\frac{h}{\pi} \int_{0}^{\pi|y| / h} \frac{\sinh t}{t} \mathrm{~d} t \\
& \leq \frac{3 h}{2 \pi} \frac{\sinh (\pi y / h)}{\pi y / h},
\end{aligned}
$$

which completes the proof.

Using this lemma, we can prove the convergence of the term $\left\|\left(\mathcal{V}-\mathcal{V}_{N}^{\mathrm{SE}}\right) \mathcal{V}_{N}^{\mathrm{SE}}\right\|_{\mathcal{L}(\mathbf{C}, \mathbf{C})}$ as described below.

Lemma 6.5. Let $k$ satisfy the assumptions in Theorem 3.4. Then, there exists a constant $C$ independent of $N$ such that

$$
\left\|\left(\mathcal{V}-\mathcal{V}_{N}^{\mathrm{SE}}\right) \mathcal{V}_{N}^{\mathrm{SE}}\right\|_{\mathcal{L}(\mathbf{C}, \mathbf{C})} \leq C h,
$$

where $h$ is the mesh size defined by (2.5).

Proof. We show that there exists a constant $C$ independent of $N$ and $f$ such that

$$
\left\|\left(\mathcal{V}-\mathcal{V}_{N}^{\mathrm{SE}}\right) \mathcal{V}_{N}^{\mathrm{SE}} f\right\|_{\mathbf{C}} \leq C\|f\|_{\mathbf{C}} h
$$

holds for all $f \in \mathbf{C}$. Let us define a function $F_{i}(t, s)$ as

$$
F_{i}(t, s)=k(t, s) k\left(s, t_{i}^{\mathrm{SE}}\right) Q\left(t_{i}^{\mathrm{SE}}\right) J(i, h)\left(\left\{\psi^{\mathrm{SE}}\right\}^{-1}(s)\right) .
$$

Then, $\left(\mathcal{V}-\mathcal{V}_{N}^{\mathrm{SE}}\right) \mathcal{V}_{N}^{\mathrm{SE}} f$ can be rewritten as

$$
\begin{aligned}
& \left(\mathcal{V}-\mathcal{V}_{N}^{\mathrm{SE}}\right) \mathcal{V}_{N}^{\mathrm{SE}}[f](t) \\
& =\sum_{i=-N}^{N} \frac{f\left(t_{i}^{\mathrm{SE}}\right)}{b-a}\left\{\int_{a}^{t} F_{i}(t, s) \mathrm{d} s-\sum_{j=-N}^{N} F_{i}\left(t, t_{j}^{\mathrm{SE}}\right)\left\{\psi^{\mathrm{SE}}\right\}^{\prime}(j h) J(j, h)\left(\left\{\psi^{\mathrm{SE}}\right\}^{-1}(t)\right)\right\} .
\end{aligned}
$$

First, we evaluate the part in $\{\cdot\}$. From $k(t, \cdot) Q(\cdot) \in \mathbf{L}_{\alpha}\left(\psi^{\mathrm{SE}}\left(\mathscr{D}_{d}\right)\right)$ and Lemma 6.4 it holds that

$$
\left|F_{i}(t, z) Q(z)\right| \leq K|Q(z)|^{\alpha} K Q^{\alpha}\left(t_{i}^{\mathrm{SE}}\right) \frac{5 h}{\pi} \frac{\sinh (\pi d / h)}{\pi d / h}
$$


for all integers $i$ and $t \in[a, b]$ and $z \in \psi^{\mathrm{SE}}\left(\mathscr{D}_{d}\right)$. Therefore, $F_{i}(t, \cdot)$ satisfies the assumptions of Theorem 2.3 , from which we have

$$
\begin{aligned}
& \left|\int_{a}^{t} F_{i}(t, s) \mathrm{d} s-\sum_{j=-N}^{N} F_{i}\left(t, t_{j}^{\mathrm{SE}}\right)\left\{\psi^{\mathrm{SE}}\right\}^{\prime}(j h) J(j, h)\left(\left\{\psi^{\mathrm{SE}}\right\}^{-1}(t)\right)\right| \\
& \leq\left\{K^{2} Q^{\alpha}\left(t_{i}^{\mathrm{SE}}\right) \frac{5 h}{\pi} \frac{\sinh (\pi d / h)}{\pi d / h}\right\}(b-a)^{2 \alpha-1} C_{\alpha, d}^{\mathrm{SE}} \mathrm{e}^{-\sqrt{\pi d \alpha N}} \\
& =\frac{5 K^{2} Q^{\alpha}\left(t_{i}^{\mathrm{SE}}\right)(b-a)^{2 \alpha-1} C_{\alpha, d}^{\mathrm{SE}}}{\pi^{2} d} \cdot h^{2}\left[\sinh (\pi d / h) \mathrm{e}^{-\pi d / h}\right] .
\end{aligned}
$$

The last equality holds from (2.5). Furthermore, $\left|\sinh (\pi d / h) \mathrm{e}^{-\pi d / h}\right| \leq 1 / 2$ holds, which implies that

$$
\begin{aligned}
\left|\left(\mathcal{V}-\mathcal{V}_{N}^{\mathrm{SE}}\right) \mathcal{V}_{N}^{\mathrm{SE}}[f](t)\right| & \leq \sum_{i=-N}^{N} \frac{\left|f\left(t_{i}^{\mathrm{SE}}\right)\right|}{b-a} \frac{5 K^{2} Q^{\alpha}\left(t_{i}^{\mathrm{SE}}\right)(b-a)^{2 \alpha-1} C_{\alpha, d}^{\mathrm{SE}}}{\pi^{2} d} \cdot \frac{h^{2}}{2} \\
& \leq\|f\|_{\mathbf{C}} \frac{5 K^{2}(b-a)^{2 \alpha-2} C_{\alpha, d}^{\mathrm{SE}}}{2 \pi^{2} d}\left\{h \sum_{i=-N}^{N} Q^{\alpha}\left(t_{i}^{\mathrm{SE}}\right)\right\} .
\end{aligned}
$$

The term in $\{\cdot\}$ is uniformly bounded since it converges to $(b-a)^{2 \alpha} \mathrm{B}(\alpha, \alpha)$. This shows the desired inequality (6.8).

Remark 6.6. In this proof we used Theorem 2.3, which states that the convergence rate is $\mathrm{O}\left(\mathrm{e}^{-\sqrt{\pi d \alpha N}}\right)$, in order to obtain (6.10). However, as stated in Remark 2.5. if we employ the existing results $\mathrm{O}\left(\sqrt{N} \mathrm{e}^{-\sqrt{\pi d \alpha N}}\right)$, we cannot prove the desired convergence, since $\left\|\left(\mathcal{V}-\mathcal{V}_{N}^{\mathrm{SE}}\right) \mathcal{V}_{N}^{\mathrm{SE}}\right\| \sim \sqrt{N} h \sim$ const. $>0$.

Thus, all conditions $1-4$ in Theorem 6.2 are fulfilled, and the next lemma follows.

Lemma 6.7. Suppose that the assumptions in Theorem 3.4 are fulfilled. Then, there exists a positive integer $N_{0}$ such that for all $N \geq N_{0}$, (6.1) has a unique solution $v \in \mathbf{C}$. Furthermore, there exists a constant $C$ independent of $N$ such that for all $N \geq N_{0}$,

$$
\|u-v\|_{\mathbf{C}} \leq C\left\|\mathcal{V} u-\mathcal{V}_{N}^{\mathrm{SE}} u\right\|_{\mathbf{C}}
$$

Proof. We have already confirmed the assumptions in Theorem 6.2, and hence, we only need to show the boundedness of the term $\left\|\left(\mathcal{I}-\mathcal{V}_{N}^{\mathrm{SE}}\right)^{-1}\right\|_{\mathcal{L}(\mathbf{C}, \mathbf{C})}$ (see (6.7)). From (6.6), the claim follows if we prove that the term $\left\|\mathcal{V}_{N}^{\mathrm{SE}}\right\|_{\mathcal{L}(\mathbf{C}, \mathbf{C})}$ is uniformly bounded. From Lemma 6.3 and $k(t, \cdot) Q(\cdot) \in \mathbf{L}_{\alpha}\left(\psi^{\mathrm{SE}}\left(\mathscr{D}_{d}\right)\right)$, we have

$$
\begin{aligned}
\left|\mathcal{V}_{N}^{\mathrm{SE}}[f](t)\right| & =\left|\sum_{j=-N}^{N} k\left(t, t_{j}^{\mathrm{SE}}\right) f\left(t_{j}^{\mathrm{SE}}\right) \frac{Q\left(t_{j}^{\mathrm{SE}}\right)}{b-a} J(j, h)\left(\left\{\psi^{\mathrm{SE}}\right\}^{-1}(t)\right)\right| \\
& \leq \frac{1.1 K\|f\|_{\mathbf{C}}}{b-a}\left\{h \sum_{j=-N}^{N} Q^{\alpha}\left(t_{j}^{\mathrm{SE}}\right)\right\}
\end{aligned}
$$

for all $f \in \mathbf{C}$. The term in $\{\cdot\}$ is uniformly bounded since it converges to $(b-$ $a)^{2 \alpha} \mathrm{B}(\alpha, \alpha)$. This completes the proof. 
6.1.4. Step 3: Proof of the exponential convergence. Now, we are in a position to prove Theorem 3.4

Proof. It only remains to evaluate the term $\left\|\mathcal{V} u-\mathcal{V}_{N}^{\mathrm{SE}} u\right\|_{\mathbf{C}}$ in (6.11). From Theorem 3.2 , we have $u \in \mathbf{H}^{\infty}\left(\psi^{\mathrm{SE}}\left(\mathscr{D}_{d}\right)\right)$. Hence, $k(t, \cdot) u(\cdot) Q(\cdot) \in \mathbf{L}_{\alpha}\left(\psi^{\mathrm{SE}}\left(\mathscr{D}_{d}\right)\right)$, and we can apply Theorem 2.3 . This gives the desired estimate.

6.2. Convergence analysis of the DE-Sinc-Nyström methods. Next, we prove the DE case, i.e., Theorem 3.5 .

6.2.1. Sketch of the proof. The proof is similar to the SE case. Let us define the operator $\mathcal{V}_{N}^{\mathrm{DE}}$ as

$$
\mathcal{V}_{N}^{\mathrm{DE}}[f](t)=\sum_{j=-N}^{N} k\left(t, t_{j}^{\mathrm{DE}}\right) f\left(t_{j}^{\mathrm{DE}}\right)\left\{\psi^{\mathrm{DE}}\right\}^{\prime}(j h) J(j, h)\left(\left\{\psi^{\mathrm{DE}}\right\}^{-1}(t)\right),
$$

which is the approximation of $\mathcal{V} f$ by the DE-Sinc indefinite integration. Then, consider the following three equations:

$$
\begin{aligned}
(\mathcal{I}-\mathcal{V}) u & =g, \\
\left(\mathcal{I}-\mathcal{V}_{N}^{\mathrm{DE}}\right) v & =g, \\
\left(I_{m}-V_{m}^{\mathrm{DE}}\right) \boldsymbol{c}_{m} & =\boldsymbol{g}_{m}^{\mathrm{DE}} .
\end{aligned}
$$

The first equation is nothing but (1.1), the second is (3.7), and the third is (3.10). In order to prove the existence of $\left(I_{m}-V_{m}^{\mathrm{DE}}\right)^{-1}$, which represents the feasibility of the scheme, we prove the following two claims:

1. Equation (6.13) is uniquely solvable if and only if (6.12) is uniquely solvable (Lemma 6.8).

2. Equation (6.12) is uniquely solvable for all sufficiently large $N$ (Lemma 6.10). Combining Lemmas 6.8 and 6.10, we conclude Theorem 3.5.

6.2.2. Step 1: Equivalence of the solvability of (6.13) and (6.12). The next lemma holds, which can be proved in exactly the same way as Lemma 6.1.

Lemma 6.8. The following statements are equivalent:

(A) Equation (6.12) has a unique solution $v \in \mathbf{C}$.

(B) Equation (6.13) has a unique solution $\boldsymbol{c}_{m} \in \mathbb{R}^{m}$.

6.2.3. Step 2: Solvability of 6.12) for all sufficiently large $N$. Our task is to show that conditions $1-4$ in Theorem 6.2 are fulfilled with $\mathcal{X}=\mathcal{V}$ and $\mathcal{X}_{n}=\mathcal{V}_{N}^{\text {DE }}$, under the assumptions in Theorem 3.5. Conditions $1-3$ clearly hold by the same argument in the SE case. Condition 4 is confirmed by the following lemma.

Lemma 6.9. Let $k$ satisfy the assumptions in Theorem 3.5. Then, there exists a constant $C$ independent of $N$ such that

$$
\left\|\left(\mathcal{V}-\mathcal{V}_{N}^{\mathrm{DE}}\right) \mathcal{V}_{N}^{\mathrm{DE}}\right\|_{\mathcal{L}(\mathbf{C}, \mathbf{C})} \leq C h^{2}
$$

where $h$ is a mesh size defined by (2.6).

Proof. We show that there exists a constant $C$ independent of $N$ and $f$ such that

$$
\left\|\left(\mathcal{V}-\mathcal{V}_{N}^{\mathrm{DE}}\right) \mathcal{V}_{N}^{\mathrm{DE}} f\right\|_{\mathbf{C}} \leq C\|f\|_{\mathbf{C}} h^{2}
$$

holds for all $f \in \mathbf{C}$. Let us define a function $F_{i}(t, s)$ as

$$
F_{i}(t, s)=k(t, s) k\left(s, t_{i}^{\mathrm{DE}}\right) Q\left(t_{i}^{\mathrm{DE}}\right) J(i, h)\left(\left\{\psi^{\mathrm{DE}}\right\}^{-1}(s)\right) .
$$


Then, $\left(\mathcal{V}-\mathcal{V}_{N}^{\mathrm{DE}}\right) \mathcal{V}_{N}^{\mathrm{DE}} f$ can be rewritten as

$$
\begin{aligned}
(\mathcal{V} & \left.-\mathcal{V}_{N}^{\mathrm{DE}}\right) \mathcal{V}_{N}^{\mathrm{DE}}[f](t) \\
= & \sum_{i=-N}^{N} \frac{f\left(t_{i}^{\mathrm{DE}}\right) \pi \cosh (i h)}{b-a} \\
& \times\left\{\int_{a}^{t} F_{i}(t, s) \mathrm{d} s-\sum_{j=-N}^{N} F_{i}\left(t, t_{j}^{\mathrm{DE}}\right)\left\{\psi^{\mathrm{DE}}\right\}^{\prime}(j h) J(j, h)\left(\left\{\psi^{\mathrm{DE}}\right\}^{-1}(t)\right)\right\} .
\end{aligned}
$$

First, we evaluate the part in $\{\cdot\}$. From $k(t, \cdot) Q(\cdot) \in \mathbf{L}_{\alpha}\left(\psi^{\mathrm{DE}}\left(\mathscr{D}_{d}\right)\right)$ and Lemma 6.4 it holds that

$$
\left|F_{i}(t, z) Q(z)\right| \leq K|Q(z)|^{\alpha} K Q^{\alpha}\left(t_{i}^{\mathrm{DE}}\right) \frac{5 h}{\pi} \frac{\sinh (\pi d / h)}{\pi d / h}
$$

for all integers $i$ and $t \in[a, b]$ and $z \in \psi^{\mathrm{DE}}\left(\mathscr{D}_{d}\right)$. Therefore, $F_{i}(t, \cdot)$ satisfies the assumptions of Theorem 2.4 from which we have

$$
\begin{aligned}
& \left|\int_{a}^{t} F_{i}(t, s) \mathrm{d} s-\sum_{j=-N}^{N} F_{i}\left(t, t_{j}^{\mathrm{DE}}\right)\left\{\psi^{\mathrm{DE}}\right\}^{\prime}(j h) J(j, h)\left(\left\{\psi^{\mathrm{DE}}\right\}^{-1}(t)\right)\right| \\
& \leq\left\{K^{2} Q^{\alpha}\left(t_{i}^{\mathrm{DE}}\right) \frac{5 h}{\pi} \frac{\sinh (\pi d / h)}{\pi d / h}\right\}(b-a)^{2 \alpha-1} C_{\alpha, d}^{\mathrm{DE}} \frac{\log (2 d N / \alpha)}{N} \exp \left[\frac{-\pi d N}{\log (2 d N / \alpha)}\right] \\
& =\frac{5 K^{2} Q^{\alpha}\left(t_{i}^{\mathrm{DE}}\right)(b-a)^{2 \alpha-1} C_{\alpha, d}^{\mathrm{DE}}}{\pi^{2} d} \cdot h^{3}\left[\sinh (\pi d / h) \mathrm{e}^{-\pi d / h}\right] .
\end{aligned}
$$

The last equality holds from (2.6) . Furthermore, $\left|\sinh (\pi d / h) \mathrm{e}^{-\pi d / h}\right| \leq 1 / 2$ holds, implying that

$$
\begin{aligned}
\left|\left(\mathcal{V}-\mathcal{V}_{N}^{\mathrm{DE}}\right) \mathcal{V}_{N}^{\mathrm{DE}}[f](t)\right| & \leq \sum_{i=-N}^{N} \frac{\left|f\left(t_{i}^{\mathrm{DE}}\right)\right| \pi \cosh (i h)}{b-a} \frac{5 K^{2} Q^{\alpha}\left(t_{i}^{\mathrm{DE}}\right)(b-a)^{2 \alpha-1} C_{\alpha, d}^{\mathrm{DE}}}{\pi^{2} d} \cdot \frac{h^{3}}{2} \\
& \leq\|f\|_{\mathbf{C}} \frac{5 K^{2}(b-a)^{2 \alpha-2} C_{\alpha, d}^{\mathrm{DE}}}{2 \pi^{2} d} h^{2}\left\{h \sum_{i=-N}^{N} \pi \cosh (i h) Q^{\alpha}\left(t_{i}^{\mathrm{DE}}\right)\right\} .
\end{aligned}
$$

The term in $\{\cdot\}$ is uniformly bounded since it converges to $(b-a)^{2 \alpha} \mathrm{B}(\alpha, \alpha)$. This shows the desired inequality (6.14).

Thus, all conditions $1-4$ in Theorem 6.2 are fulfilled, and the next lemma follows.

Lemma 6.10. Suppose that the assumptions in Theorem 3.5 are fulfilled. Then, there exists a positive integer $N_{0}$ such that for all $N \geq N_{0}$, (6.12) has a unique solution $v \in \mathbf{C}$. Furthermore, there exists a constant $C$ independent of $N$ such that for all $N \geq N_{0}$,

$$
\|u-v\|_{\mathbf{C}} \leq C\left\|\mathcal{V} u-\mathcal{V}_{N}^{\mathrm{DE}} u\right\|_{\mathbf{C}}
$$

Proof. We have already confirmed the assumptions in Theorem 6.2, and it only remains to show the boundedness of the term $\left\|\left(\mathcal{I}-\mathcal{V}_{N}^{\mathrm{DE}}\right)^{-1}\right\|_{\mathcal{L}(\mathbf{C}, \mathbf{C})}$ (see (6.7)). From (6.6), the claim follows if we prove that the term $\left\|\mathcal{V}_{N}^{\mathrm{DE}}\right\|_{\mathcal{L}(\mathbf{C}, \mathbf{C})}$ is uniformly 
bounded. From Lemma 6.3 and $k(t, \cdot) Q(\cdot) \in \mathbf{L}_{\alpha}\left(\psi^{\mathrm{DE}}\left(\mathscr{D}_{d}\right)\right)$, we have

$$
\begin{aligned}
\left|\mathcal{V}_{N}^{\mathrm{DE}}[f](t)\right| & =\left|\sum_{j=-N}^{N} k\left(t, t_{j}^{\mathrm{DE}}\right) f\left(t_{j}^{\mathrm{DE}}\right) \frac{\pi \cosh (j h) Q\left(t_{j}^{\mathrm{DE}}\right)}{b-a} J(j, h)\left(\left\{\psi^{\mathrm{DE}}\right\}^{-1}(t)\right)\right| \\
& \leq \frac{1.1 K\|f\|_{\mathbf{C}}}{b-a}\left\{h \sum_{j=-N}^{N} \pi \cosh (j h) Q^{\alpha}\left(t_{j}^{\mathrm{DE}}\right)\right\}
\end{aligned}
$$

for all $f \in \mathbf{C}$. The term in $\{\cdot\}$ is uniformly bounded since it converges to $(b-$ $a)^{2 \alpha} \mathrm{B}(\alpha, \alpha)$. This completes the proof.

6.2.4. Step 3: Proof of the exponential convergence. Now, we are in a position to prove Theorem 3.5 .

Proof. It remains to evaluate the term $\left\|\mathcal{V} u-\mathcal{V}_{N}^{\mathrm{DE}} u\right\|_{\mathbf{C}}$ in 6.16). From Theorem 3.2. we have $u \in \mathbf{H}^{\infty}\left(\psi^{\mathrm{DE}}\left(\mathscr{D}_{d}\right)\right)$. Hence, $k(t, \cdot) u(\cdot) Q(\cdot) \in \mathbf{L}_{\alpha}\left(\psi^{\mathrm{DE}}\left(\mathscr{D}_{d}\right)\right)$, and we can apply Theorem 2.4. Thus, this theorem is established.

\section{Proof of MAin Result 3}

We prove only Theorem 3.7 (Theorem 3.8 can be proved in exactly the same way by replacing 'SE' with 'DE').

Proof. If we show the following two inequalities,

$$
\begin{aligned}
\left\|I_{m}-V_{m}^{\mathrm{SE}}\right\|_{\infty} & \leq\left\|\mathcal{I}-\mathcal{V}_{N}^{\mathrm{SE}}\right\|_{\mathcal{L}(\mathbf{C}, \mathbf{C})} \\
\left\|\left(I_{m}-V_{m}^{\mathrm{SE}}\right)^{-1}\right\|_{\infty} & \leq\left\|\left(\mathcal{I}-\mathcal{V}_{N}^{\mathrm{SE}}\right)^{-1}\right\|_{\mathcal{L}(\mathbf{C}, \mathbf{C})},
\end{aligned}
$$

then Theorem 3.7 is established. The reason is that, as proved in Lemma 6.7. $\left\|\mathcal{V}_{N}^{\mathrm{SE}}\right\|_{\mathcal{L}(\mathbf{C}, \mathbf{C})}$ and $\left\|\left(\mathcal{I}-\mathcal{V}_{N}^{\mathrm{SE}}\right)^{-1}\right\|_{\mathcal{L}(\mathbf{C}, \mathbf{C})}$ are uniformly bounded.

We show (7.1) first. For a given arbitrary $\boldsymbol{c}_{m}=\left[c_{-N}, \ldots, c_{N}\right]^{\mathrm{T}}$, choose $\gamma \in \mathbf{C}$ with the properties

$$
\|\gamma\|_{\mathbf{C}}=\left\|\boldsymbol{c}_{m}\right\|_{\infty} \quad \text { and } \quad \gamma\left(t_{i}^{\mathrm{SE}}\right)=c_{i} \quad(i=-N, \ldots, N) .
$$

Let us define a function $f$ and a vector $\boldsymbol{f}_{m}^{\mathrm{SE}}$ as

$$
f=\left(\mathcal{I}-\mathcal{V}_{N}^{\mathrm{SE}}\right) \gamma, \quad \boldsymbol{f}_{m}^{\mathrm{SE}}=\left[f\left(t_{-N}^{\mathrm{SE}}\right), \ldots, f\left(t_{N}^{\mathrm{SE}}\right)\right]^{\mathrm{T}} .
$$

Then, we have

$$
\begin{aligned}
\left\|\left(I_{m}-V_{m}^{\mathrm{SE}}\right) \boldsymbol{c}_{m}\right\|_{\infty} & =\left\|\boldsymbol{f}_{m}^{\mathrm{SE}}\right\|_{\infty} \\
& \leq\|f\|_{\mathbf{C}} \\
& =\left\|\left(\mathcal{I}-\mathcal{V}_{N}^{\mathrm{SE}}\right) \gamma\right\|_{\mathbf{C}} \\
& \leq\left\|\left(\mathcal{I}-\mathcal{V}_{N}^{\mathrm{SE}}\right)\right\|_{\mathcal{L}(\mathbf{C}, \mathbf{C})}\|\gamma\|_{\mathbf{C}} \\
& =\left\|\left(\mathcal{I}-\mathcal{V}_{N}^{\mathrm{SE}}\right)\right\|_{\mathcal{L}(\mathbf{C}, \mathbf{C})}\left\|\boldsymbol{c}_{m}\right\|_{\infty},
\end{aligned}
$$

which shows (7.1).

Next, we show (7.2). Note that $\left(\mathcal{I}-\mathcal{V}_{N}^{\mathrm{SE}}\right)^{-1}$ exists for all sufficiently large $N$ from Lemma 6.7, and from Lemma 6.1, $\left(I_{m}-V_{m}^{\mathrm{SE}}\right)^{-1}$ always exists if $\left(\mathcal{I}-\mathcal{V}_{N}^{\mathrm{SE}}\right)^{-1}$ exists. For a given arbitrary $\boldsymbol{c}_{m}=\left[c_{-N}, \ldots, c_{N}\right]^{\mathrm{T}}$, choose $\gamma \in \mathbf{C}$ with the properties (7.3). Furthermore, define a function $f$ and a vector $\boldsymbol{f}_{m}^{\mathrm{SE}}$ as

$$
f=\left(\mathcal{I}-\mathcal{V}_{N}^{\mathrm{SE}}\right)^{-1} \gamma, \quad \boldsymbol{f}_{m}^{\mathrm{SE}}=\left[f\left(t_{-N}^{\mathrm{SE}}\right), \ldots, f\left(t_{N}^{\mathrm{SE}}\right)\right]^{\mathrm{T}} .
$$


Then, we have

$$
\begin{aligned}
\left\|\left(I_{m}-V_{m}^{\mathrm{SE}}\right)^{-1} \boldsymbol{c}_{m}\right\|_{\infty} & =\left\|\boldsymbol{f}_{m}^{\mathrm{SE}}\right\|_{\infty} \\
& \leq\|f\|_{\mathbf{C}} \\
& =\left\|\left(\mathcal{I}-\mathcal{V}_{N}^{\mathrm{SE}}\right)^{-1} \gamma\right\|_{\mathbf{C}} \\
& \leq\left\|\left(\mathcal{I}-\mathcal{V}_{N}^{\mathrm{SE}}\right)^{-1}\right\|_{\mathcal{L}(\mathbf{C}, \mathbf{C})}\|\gamma\|_{\mathbf{C}} \\
& =\left\|\left(\mathcal{I}-\mathcal{V}_{N}^{\mathrm{SE}}\right)^{-1}\right\|_{\mathcal{L}(\mathbf{C}, \mathbf{C})}\left\|\boldsymbol{c}_{m}\right\|_{\infty},
\end{aligned}
$$

which shows (7.2).

\section{RESUlts FOR EQUATIONS OF THE FIRST KIND}

In this section, we consider the schemes developed by Muhammad et al. 14 for Volterra integral equations of the first kind (1.8). Their idea is simply to reduce (1.8) to (1.1) as follows. If both $k(\cdot, s)$ and $g$ are differentiable, (1.8) can be reduced to

$$
k(t, t) u(t)+\int_{a}^{t} \frac{\partial k(t, s)}{\partial t} u(s) \mathrm{d} s=g^{\prime}(t)
$$

and if $k(t, t) \neq 0$, it can be rewritten as second kind equation

$$
u(t)-\int_{a}^{t} \tilde{k}(t, s) u(s) \mathrm{d} s=\tilde{g}(t)
$$

where

$$
\tilde{k}(t, s)=-\frac{1}{k(t, t)} \frac{\partial k(t, s)}{\partial t}, \quad \tilde{g}(t)=\frac{g^{\prime}(t)}{k(t, t)} .
$$

Even when $k(t, t)=0$, if the new kernel in (8.1) satisfies $\partial k(t, t) / \partial t \neq 0$, then we can get a second kind equation by one more differentiation. Although such an attempt can be repeated as many times as desired, here, let us assume $k(t, t) \neq 0$ and consider (8.2) for simplicity (in the same manner as Muhammad et al. 14]).

Once we get the second kind equation (8.2), we can apply the results in Section 3 , In Sections 8.1 and 8.2 , we review the schemes and theorem given by Muhammad et al. 14. Then, we state the three main results in Section 8.3. Their proofs are omitted, since the results follow from the proofs for second kind equations described in Sections 3.3 3.5.

8.1. SE-Sinc-Nyström method. Let us consider the SE case first. Let $u \in$ $\mathbf{H}^{\infty}\left(\psi^{\mathrm{SE}}\left(\mathscr{D}_{d}\right)\right)$ and $\tilde{k}(t, \cdot) Q(\cdot) \in \mathbf{L}_{\alpha}\left(\psi^{\mathrm{SE}}\left(\mathscr{D}_{d}\right)\right)$ for all $t \in[a, b]$, and consider the approximated equation

$$
\tilde{u}_{N}^{\mathrm{SE}}(t)=\tilde{g}(t)+\sum_{j=-N}^{N} \tilde{k}\left(t, t_{j}^{\mathrm{SE}}\right) \tilde{u}_{N}^{\mathrm{SE}}\left(t_{j}^{\mathrm{SE}}\right)\left\{\psi^{\mathrm{SE}}\right\}^{\prime}(j h) J(j, h)\left(\left\{\psi^{\mathrm{SE}}\right\}^{-1}(t)\right) .
$$

By discretizing this equation at the SE-Sinc points (3.3), we have a linear equation

$$
\left(I_{m}-\tilde{V}_{m}^{\mathrm{SE}}\right) \tilde{\boldsymbol{u}}_{m}^{\mathrm{SE}}=\tilde{\boldsymbol{g}}_{m}^{\mathrm{SE}},
$$

where $\tilde{\boldsymbol{u}}_{m}^{\mathrm{SE}}, \tilde{V}_{m}^{\mathrm{SE}}$, and $\tilde{\boldsymbol{g}}_{m}^{\mathrm{SE}}$ are defined as in (3.2), (3.5), and (3.6), respectively. By solving (8.5), the approximated solution $\tilde{u}_{N}^{\mathrm{SE}}$ is determined by (8.4). 
8.2. DE-Sinc-Nyström method. Next, let us consider the DE case. Let $u \in$ $\mathbf{H}^{\infty}\left(\psi^{\mathrm{DE}}\left(\mathscr{D}_{d}\right)\right)$ and $\tilde{k}(t, \cdot) Q(\cdot) \in \mathbf{L}_{\alpha}\left(\psi^{\mathrm{DE}}\left(\mathscr{D}_{d}\right)\right)$ for all $t \in[a, b]$, and consider the approximated equation

$$
\tilde{u}_{N}^{\mathrm{DE}}(t)=\tilde{g}(t)+\sum_{j=-N}^{N} \tilde{k}\left(t, t_{j}^{\mathrm{DE}}\right) \tilde{u}_{N}^{\mathrm{DE}}\left(t_{j}^{\mathrm{DE}}\right)\left\{\psi^{\mathrm{DE}}\right\}^{\prime}(j h) J(j, h)\left(\left\{\psi^{\mathrm{DE}}\right\}^{-1}(t)\right) .
$$

By discretizing this equation at the DE-Sinc points (3.9), we have a linear equation

$$
\left(I_{m}-\tilde{V}_{m}^{\mathrm{DE}}\right) \tilde{\boldsymbol{u}}_{m}^{\mathrm{DE}}=\tilde{\boldsymbol{g}}_{m}^{\mathrm{DE}},
$$

where $\tilde{\boldsymbol{u}}_{m}^{\mathrm{DE}}, \tilde{V}_{m}^{\mathrm{DE}}$, and $\tilde{\boldsymbol{g}}_{m}^{\mathrm{DE}}$ are defined as in (3.8), (3.11), and (3.12), respectively. By solving (8.7), the approximated solution $\tilde{u}_{N}^{\mathrm{DE}}$ is determined by (8.6).

For this scheme (DE-Sinc-Nyström method), Muhammad et al. 14] obtained the following error analysis.

Theorem 8.1 (Muhammad et al. 14, Theorem 3.3]). Let $u$ be the solution of (1.8). Let $u \in \mathbf{H}^{\infty}\left(\psi^{\mathrm{DE}}\left(\mathscr{D}_{d}\right)\right)$, let $k(t, t) \neq 0$, let $g(t)$ be differentiable, and let $\{\partial k(t, \cdot) / \partial t\} Q(\cdot) \in \mathbf{L}_{\alpha}\left(\psi^{\mathrm{DE}}\left(\mathscr{D}_{d}\right)\right)$ for all $t \in[a, b]$. Then, there exist constants $C$ and $C^{\prime}$ independent of $N$ such that

$$
\sup _{a<t<b}\left|u(t)-\tilde{u}_{N}^{\mathrm{DE}}(t)\right| \leq\left(C \sqrt{N}\left\|\left(I_{m}-\tilde{V}_{m}^{\mathrm{DE}}\right)^{-1}\right\|_{2}+C^{\prime}\right) \frac{\log N}{N} \exp \left[\frac{-\pi d N}{\log (2 d N / \alpha)}\right] \text {. }
$$

8.3. New theoretical results for equations of the first kind. The result corresponding to Section 3.3 (estimating the tuning parameter $d$ ) is described below.

Theorem 8.2. Let $\mathscr{D}=\psi^{\mathrm{SE}}\left(\mathscr{D}_{d}\right)$ or $\mathscr{D}=\psi^{\mathrm{DE}}\left(\mathscr{D}_{d}\right)$. Let $g^{\prime} \in \mathbf{H}^{\infty}(\mathscr{D})$, let $\{\partial k(z, \cdot) / \partial z\} Q(\cdot) \in \mathbf{L}_{\alpha}(\mathscr{D})$, and let $\{\partial k(\cdot, w) / \partial z\} Q(w) \in \mathbf{H}^{\infty}(\mathscr{D})$ for all $z, w \in$ $\mathscr{D}$. Furthermore, let $1 / k(z, z) \in \mathbf{H}^{\infty}(\mathscr{D})$. Then, (1.8) has a unique solution $u \in \mathbf{H}^{\infty}(\mathscr{D})$.

The results corresponding to Section 3.4 (proofs of the exponential convergence) are the following theorems.

Theorem 8.3. Let the assumptions in Theorem 8.2 be satisfied with $\mathscr{D}=\psi^{\mathrm{SE}}\left(\mathscr{D}_{d}\right)$. Let $g^{\prime} \in C([a, b])$, let $\{\partial k(t, \cdot) / \partial t\} Q(\cdot) \in C([a, b])$, and let $\{\partial k(\cdot, s) / \partial t\} Q(s) \in$ $C([a, b])$ for all $t, s \in[a, b]$. Furthermore, let $1 / k(t, t) \in C([a, b])$. Then, there exists a positive integer $N_{0}$ such that for all $N \geq N_{0}$, the coefficient matrix $\left(I_{m}-\right.$ $\left.\tilde{V}_{m}^{\mathrm{SE}}\right)$ is invertible. Furthermore, there exists a constant $C$ for all $N \geq N_{0}$ such that

$$
\max _{a \leq t \leq b}\left|u(t)-\tilde{u}_{N}^{\mathrm{SE}}(t)\right| \leq C \mathrm{e}^{-\sqrt{\pi d \alpha N}} .
$$

Theorem 8.4. Let the assumptions in Theorem 8.2 be satisfied with $\mathscr{D}=\psi^{\mathrm{DE}}\left(\mathscr{D}_{d}\right)$. Let $g^{\prime} \in C([a, b])$, let $\{\partial k(t, \cdot) / \partial t\} Q(\cdot) \in C([a, b])$, and let $\{\partial k(\cdot, s) / \partial t\} Q(s) \in$ $C([a, b])$ for all $t, s \in[a, b]$. Furthermore, let $1 / k(t, t) \in C([a, b])$. Then, there exists a positive integer $N_{0}$ such that for all $N \geq N_{0}$, the coefficient matrix $\left(I_{m}-\right.$ $\left.\tilde{V}_{m}^{\mathrm{DE}}\right)$ is invertible. Furthermore, there exists a constant $C$ for all $N \geq N_{0}$ such that

$$
\max _{a \leq t \leq b}\left|u(t)-\tilde{u}_{N}^{\mathrm{DE}}(t)\right| \leq C \frac{\log (2 d N / \alpha)}{N} \exp \left[\frac{-\pi d N}{\log (2 d N / \alpha)}\right] .
$$

Furthermore, the results corresponding to Section 3.5 (analyses of conditions of the resulting systems) are also given as follows. 
Theorem 8.5. Let the assumptions in Theorem 8.3 be satisfied. Then, there exists a constant $C_{1}$ independent of $N$ such that

$$
\left\|\left(I_{m}-\tilde{V}_{m}^{\mathrm{SE}}\right)\right\|_{\infty} \leq C_{1} .
$$

Furthermore, there exists a constant $C_{2}$ independent of $N$ such that for all $N \geq N_{0}$,

$$
\left\|\left(I_{m}-\tilde{V}_{m}^{\mathrm{SE}}\right)^{-1}\right\|_{\infty} \leq C_{2},
$$

where $N_{0}$ is the same integer as in Theorem 8.3 .

Theorem 8.6. Let the assumptions in Theorem 8.4 be satisfied. Then, there exists a constant $C_{1}$ independent of $N$ such that

$$
\left\|\left(I_{m}-\tilde{V}_{m}^{\mathrm{DE}}\right)\right\|_{\infty} \leq C_{1} .
$$

Furthermore, there exists a constant $C_{2}$ independent of $N$ such that for all $N \geq N_{0}$,

$$
\left\|\left(I_{m}-\tilde{V}_{m}^{\mathrm{DE}}\right)^{-1}\right\|_{\infty} \leq C_{2},
$$

where $N_{0}$ is the same integer as in Theorem 8.4 .

\section{Concluding Remarks}

In this paper, the Sinc-Nyström methods for (1.1) and (1.8) developed by Muhammad et al. 14 were considered, and the following new theoretical results were obtained: 1) a way to estimate a tuning parameter $d, 2$ ) rigorous proof of the exponential convergence of the schemes, and 3) proof that the resulting system is well conditioned. These results were established for both the SE- and DE-Sinc-Nyström methods. These results were also confirmed by numerical experiments.

On the basis of these results, we can analyze the Sinc-collocation methods for Volterra integral equations [19, which will be reported in a future study.

\section{REFERENCES}

[1] P. M. Anselone, Collectively Compact Operator Approximation Theory and Applications to Integral Equations, Prentice-Hall Inc., Englewood Cliffs, N. J., 1971. With an appendix by Joel Davis; Prentice-Hall Series in Automatic Computation. MR0443383 (56 \#1753)

[2] K. E. Atkinson, The Numerical Solution of Integral Equations of the Second Kind, Cambridge Monographs on Applied and Computational Mathematics, vol. 4, Cambridge University Press, Cambridge, 1997. MR1464941 (99d:65364)

[3] R. Bellman and K. L. Cooke, Differential-difference Equations, Academic Press, New York, 1963. MR0147745(26 \#5259)

[4] F. Brauer and C. Castillo-Chávez, Mathematical Models in Population Biology and Epidemiology, Texts in Applied Mathematics, vol. 40, Springer-Verlag, New York, 2001. MR.1822695 (2002k:92001)

[5] H. Brunner, Collocation Methods for Volterra Integral and Related Functional Differential Equations, Cambridge Monographs on Applied and Computational Mathematics, vol. 15, Cambridge University Press, Cambridge, 2004. MR2128285 (2005k:65002)

[6] S. Haber, Two formulas for numerical indefinite integration, Math. Comp. 60 (1993), no. 201, 279-296, DOI 10.2307/2153166. MR.1149292 (93d:65026)

[7] S. I. Kabanikhin and A. Lorenzi, Identification Problems of Wave Phenomena: Theory and Numerics, Inverse and Ill-posed Problems Series, VSP, Utrecht, 1999. MR.1741782 (2002c:65002)

[8] R. Kress, Linear Integral Equations, 2nd ed., Applied Mathematical Sciences, vol. 82, Springer-Verlag, New York, 1999. MR1723850 (2000h:45001)

[9] P. K. Kythe and P. Puri, Computational Methods for Linear Integral Equations, Birkhäuser Boston Inc., Boston, MA, 2002. MR1902732(2003e:65242) 
[10] P. K. Lamm, A survey of regularization methods for first-kind Volterra equations, Surveys on Solution Methods for Inverse Problems, Springer, Vienna, 2000, pp. 53-82. MR1766739 (2001b:65148)

[11] P. Linz, Analytical and Numerical Methods for Volterra Equations, SIAM Studies in Applied Mathematics, vol. 7, Society for Industrial and Applied Mathematics (SIAM), Philadelphia, PA, 1985. MR796318 (86m:65163)

[12] R. K. Miller and A. Unterreiter, Switching behavior of PN-diodes: Volterra integral equation models, J. Integral Equations Appl. 4 (1992), no. 2, 257-272, DOI 10.1216/jiea/1181075684. MR.1172892(93f:94030)

[13] M. Muhammad and M. Mori, Double exponential formulas for numerical indefinite integration, J. Comput. Appl. Math. 161 (2003), no. 2, 431-448, DOI 10.1016/j.cam.2003.05.002. MR2017024 (2004j:26015)

[14] M. Muhammad, A. Nurmuhammad, M. Mori, and M. Sugihara, Numerical solution of integral equations by means of the Sinc collocation method based on the double exponential transformation, J. Comput. Appl. Math. 177 (2005), no. 2, 269-286, DOI 10.1016/j.cam.2004.09.019. MR:2125318(2005m:65314)

[15] T. Okayama, T. Matsuo, and M. Sugihara, Sinc-collocation methods for weakly singular Fredholm integral equations of the second kind, J. Comput. Appl. Math. 234 (2010), no. 4, 1211-1227, DOI 10.1016/j.cam.2009.07.049. MR2609575(2011d:65421)

[16] T. Okayama, T. Matsuo, and M. Sugihara, Improvement of a sinc-collocation method for Fredholm integral equations of the second kind, BIT 51 (2011), no. 2, 339-366, DOI 10.1007/s10543-010-0289-x. MR2806534 (2012f:65227)

[17] T. Okayama, T. Matsuo, and M. Sugihara, Error estimates with explicit constants for Sinc approximation, Sinc quadrature and Sinc indefinite integration, Numer. Math. 124 (2013), no. 2, 361-394, DOI 10.1007/s00211-013-0515-y. MR.3054356

[18] A. D. Polyanin and A. V. Manzhirov, Handbook of Integral Equations, 2nd ed., Chapman \& Hall/CRC, Boca Raton, FL, 2008. MR2404728 (2009a:45001)

[19] J. Rashidinia and M. Zarebnia, Solution of a Volterra integral equation by the sinc-collocation method, J. Comput. Appl. Math. 206 (2007), no. 2, 801-813, DOI 10.1016/j.cam.2006.08.036. MR2333714 (2008d:65158)

[20] W. Rudin, Real and Complex Analysis, 3rd ed., McGraw-Hill Book Co., New York, 1987. MR924157 (88k:00002)

[21] F. Stenger, Numerical Methods Based on Sinc and Analytic Functions, Springer Series in Computational Mathematics, vol. 20, Springer-Verlag, New York, 1993. MR 1226236 (94k:65003)

[22] F. Stenger, Summary of Sinc numerical methods, J. Comput. Appl. Math. 121 (2000), no. 12, 379-420, DOI 10.1016/S0377-0427(00)00348-4. Numerical analysis in the 20th century, Vol. I, Approximation theory. MR 1780056 (2001d:65018)

[23] K. Tanaka, M. Sugihara, and K. Murota, Function classes for successful DE-Sinc approximations, Math. Comp. 78 (2009), no. 267, 1553-1571, DOI 10.1090/S0025-5718-08-02196-0. MR2501063(2010c:65019)

Graduate School of Economics, Hitotsubashi University, 2-1, Naka, Kunitachi, Tokyo, 186-8601, JAPAN

E-mail address: tokayama@econ.hit-u.ac.jp

Graduate School of Information Science and Technology, The University of Tokyo,

7-3-1, Hongo, Bunkyo, TOKYo, 113-8656, JAPAN

E-mail address: matsuo@mist.i.u-tokyo.ac.jp

Graduate School of Information Science and Technology, The University of Tokyo,

7-3-1, Hongo, Bunkyo, TOKYo, 113-8656, JAPAN

E-mail address: m_sugihara@ist.i.u-tokyo.ac.jp 